\title{
Timing Error Detector Design and Analysis for Orthogonal Space-Time Block Code Receivers
}

\author{
Pawel A. Dmochowski, Member, IEEE, and Peter J. McLane, Life Fellow, IEEE
}

\begin{abstract}
A general framework for the design of low complexity timing error detectors (TEDs) for orthogonal space-time block code (OSTBC) receivers is proposed. Specifically, we derive sufficient conditions for a difference-of-threshold-crossings timing error estimate to be robust to channel fading. General expressions for the $\mathrm{S}$-curve, estimation error variance and the signal-to-noise ratio are also obtained. As the designed detectors inherently depend on the properties of the OSTBC under consideration, we derive and evaluate the properties of TEDs for a number of known codes. Simulations are used to assess the system performance with the proposed timing detectors incorporated into the receiver timing loop operating in tracking mode. While the theoretical derivations assume a receiver with perfect channel state information and symbol decisions, simulation results include performance for pilot-symbol-based channel estimation and data symbol detection errors. For the case of frequency-flat Rayleigh fading and QPSK modulation, symbol-error-rate results show timing synchronization loss of less than $0.3 \mathrm{~dB}$ for practical timing offsets. In addition it is shown that the receiver is able to track timing drift with a normalized bandwidth of up to 0.001 .
\end{abstract}

Index Terms-Synchronization, symbol timing estimation, timing error tracking, orthogonal space-time block coding.

\section{INTRODUCTION}

$\mathbf{R}$ ECENTLY, a significant amount of research has been devoted to multiple-input multiple-output (MIMO) communication systems. In particular, orthogonal space-time block coding (OSTBC) has received a lot of attention since its development [1]-[3], due to the ability to provide excellent performance in fading while maintaining low decoding complexity. It has been recognized that the estimation of reference parameters, such as timing epoch and channel fading samples, is critical to the performance of MIMO receivers. Timing acquisition in space-time coded modems was first addressed in [4], where the receiver obtains timing information by maximizing the oversampled log-likelihood function (LLF) derived from an orthogonal training sequence. A number of improvements of this maximum likelihood (ML)-based method have subsequently been proposed, with the focus on the reduction in algorithm complexity and oversampling requirement [5], [6].

In contrast to the training-based timing acquisition methods in [4]-[6], this paper focuses on the problem of timing

Paper approved by H. Minn, the Editor for Synchronization and Equalization of the IEEE Communications Society. Manuscript received May 9, 2006; revised March 6, 2007 and August 30, 2007. This work was supported by NSERC of Canada and the Bell Mobility / Samsung Grant on Smart Antennas at Queen's University.

P. A. Dmochowski is with the Faculty of Engineering, Victoria University of Wellington, New Zealand (e-mail: pdmochowski@ieee.org).

P. J. McLane is with the Department of Electrical and Computer Engineering, Queen's University at Kingston.

Digital Object Identifier 10.1109/TCOMM.2008.060290 error tracking by means of very low complexity timing error detectors (TEDs). In a pulse-shaped system, the presence of timing errors results in intersymbol interference (ISI)corrupted data strobes. In the case of a single antenna ISI channel, it was shown [7] that a timing error measurement (TEM) in the form of a difference-of-threshold-crossings can be obtained by simple manipulation of receiver sample strobes and data symbols. The resulting TEM is then used in a timing loop for timing error tracking. In the sequel we demonstrate that in a frequency-flat Rayleigh fading environment manipulating OSTBC system matrices can result in a timing error measurement similar to that considered in [7] ${ }^{1}$. We present a general framework for the design of TEDs for OSTBC receivers, deriving sufficient conditions for TEMs robust to channel fading. In contrast to ML-based optimization techniques, which require computationally intensive likelihood function estimation followed by the search for its maximum, estimators presented here offer very low complexity as they are based on linear combining of their inputs. The designs easily lend themselves for analysis. Specifically, under the assumption of receiver channel state information (CSIR) and perfect data decisions, we derive analytical expressions for the detector S-curve, the estimation error variance and the TED signal-to-noise ratio (SNR). Examples of TEDs for some known OSTBCs are also presented. The ideal assumptions of channel and data knowledge are removed when evaluating the overall system performance by means of simulations.

The remainder of this paper is organized as follows. We begin with a system overview in Section II. The details of timing error detector design are covered in Section III, which includes the derivation of the S-curve in Section III-A, variance and SNR properties in Section III-B and examples of TEDs for particular OSTBCs in Section III-C. The analytical results are confirmed by means of simulations in Section IV. System simulations are discussed in Section V, where symbolerror-rate (SER) and timing drift bandwidth range results are presented. We conclude with a summary of findings in Section VI.

\section{SyStem OVERVIEW}

We consider a communication system comprising of $N_{t}$ transmit and $N_{r}$ receive antennas employing orthogonal spacetime block coding [1]-[3]. The transmitter encodes $N_{s}$ information symbols and transmits them over $N_{t}$ antennas in $N_{c}$ time slots, resulting in a code rate of $R=N_{s} / N_{c}$. We denote the $l$ th $N_{t} \times N_{c}$ code block by $\mathbf{X}_{l}$ and its $(i, k)$ th

\footnotetext{
${ }^{1}$ A less general approach, one limited to the Alamouti OSTBC, was given in [8] and further analyzed in [9].
} 


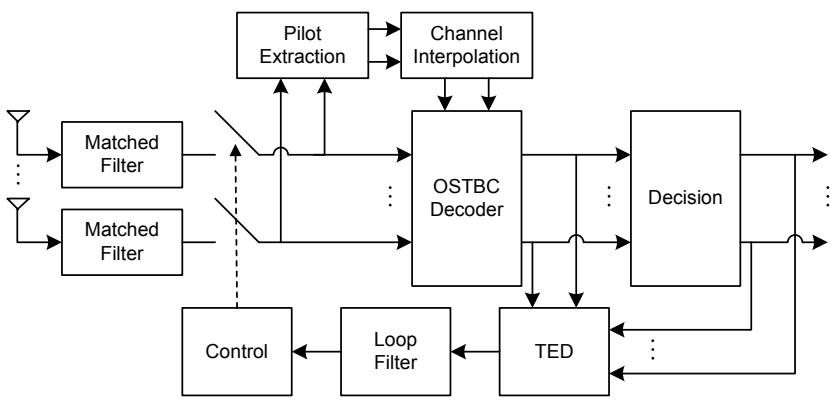

Fig. 1. Receiver diagram.

entry by ${ }^{2} x_{i}\left[l N_{c}+k\right]$. Note that $l$ is the code block index, $k=0, \ldots, N_{c}-1$ is the time slot index within the block and $i=1, \ldots, N_{t}$ is the transmit antenna index. Let the $m$ th information symbol used to encode block $\mathbf{X}_{l}$ be $a_{m, l}$, where $m=0, \ldots, N_{s}-1$. Then, using the code matrix approach of [10], $\mathbf{X}_{l}$ is given by the linear combination of $a_{m, l}$ and their conjugates

$$
\mathbf{X}_{l}=\sum_{m=0}^{N_{s}-1}\left(\Re\left\{a_{m, l}\right\} \mathbf{A}_{m}+i \Im\left\{a_{m, l}\right\} \mathbf{B}_{m}\right)
$$

where the operators $\Re\{\cdot\}$ and $\Im\{\cdot\}$ return the real and imaginary parts of their arguments, respectively, and $\mathbf{A}_{m}$ and $\mathbf{B}_{m}$ are integer code matrices of dimension $N_{t} \times N_{c}$. The TED design procedure presented here utilizes matrices $\mathbf{A}_{m}$ and $\mathbf{B}_{m}$, which can be obtained for a given OSTBC using (1), and given in [11]. The pulse shaping is split between the transmitter and the receiver, each using a root raised cosine (RRC) filter denoted by $\tilde{g}(t)$. The combined Nyquist raised cosine pulse is represented by $g(t)=\tilde{g}(t) * \tilde{g}(t)$, where $*$ denotes convolution. We assume a frequency-flat Rayleigh fading channel modeled by a $N_{r} \times N_{t}$ matrix $\mathbf{H}$. Its components, denoted by $h_{j i}$, correspond to the channel state from $i$ th transmit to $j$ th receive antenna and are assumed to be independent and identically distributed (iid), and quasi-static, that is $h_{j i}\left[l N_{c}\right] \approx h_{j i}\left[(l+1) N_{c}-1\right]$. Clarke's 2-D isotropic scattering model [12] is assumed and thus the autocorrelation of $h_{j i}$ (for all $i$ and $j$ ) is given by [13]

$$
R_{h}(\xi)=\sigma_{h}^{2} J_{0}\left(2 \pi f_{D} \xi\right)
$$

where $\sigma_{h}^{2}$ is the variance of the random process and $J_{0}(x)$ denotes the Bessel function of the first kind of order zero. The quantity $f_{D}$ in (2) denotes the maximum Doppler frequency which is assumed to be known.

The receiver diagram is given in Fig. 1. The received signal at antenna $j$ is given by

$$
r_{j}(t)=\sum_{i=1}^{N_{t}} h_{j i}(t) \sum_{n^{\prime}} x_{i}\left[n^{\prime}\right] \tilde{g}\left(t-n^{\prime} T-\tau\right)+\tilde{\eta}_{j}(t)
$$

where $x_{i}\left[n^{\prime}\right]$ is the encoded symbol transmitted by antenna $i$ for time slot $n^{\prime}=l N_{c}+k$ and $\tilde{\eta}_{j}(t)$ is a zero mean complex Gaussian noise with variance $\sigma_{\tilde{\eta}}^{2}=N_{0} / 2$ per signal

\footnotetext{
${ }^{2}$ We will differentiate between discrete and continuous quantities by placing their arguments within square brackets or parenthesis, respectively (e.g., $x(t)$ or $x[n]=x(n T))$.
}

dimension. Received signal time delay is denoted in (3) by $\tau$. After matched filtering, the signal $y_{j}(t)=r_{j}(t) * \tilde{g}(t)$ is sampled at time instants $t_{n}=n T+\hat{\tau}$, where $\hat{\tau}$ is the timing correction applied at the receiver. The residual timing error is thus given by $\epsilon=\tau-\hat{\tau}$. Due to the assumption of quasistatic fading, we have that $h_{j i}\left(t_{n}\right) \approx h_{j i}(n T)=h_{j i}[n]$ and the resulting samples are given by

$$
y_{j}[n]=\sum_{i=1}^{N_{t}} h_{j i}[n] \sum_{n^{\prime}} x_{i}\left[n^{\prime}\right] g\left(n T-n^{\prime} T+\epsilon\right)+\eta_{j}[n]
$$

where $\eta_{j}[n]$ denotes the samples of the matched filtered noise, $\eta_{j}(t)=\tilde{\eta}_{j}(t) * \tilde{g}(t)$, which are uncorrelated if sampled at the symbol rate [4]. We can re-write (4) as

$$
y_{j}[n]=\sum_{i=1}^{N_{t}} h_{j i}[n] x_{i}^{\epsilon}[n]+\eta_{j}[n]
$$

where $x_{i}^{\epsilon}[n]$ are the ISI-equivalent encoded symbols given a sampling error $\epsilon$,

$$
x_{i}^{\epsilon}[n]=\sum_{n^{\prime}} x_{i}\left[n^{\prime}\right] g\left(n T-n^{\prime} T+\epsilon\right) .
$$

Consider the samples for $n=l N_{c}, \ldots,(l+1) N_{c}-1$, corresponding to time slots $k=0, \ldots, N_{c}-1$ within a single code block $l$. We can express the output samples in (5) by a $N_{r} \times N_{c}$ matrix $\mathbf{Y}_{l}$,

$$
\mathbf{Y}_{l}=\mathbf{H}_{l} \mathbf{X}_{\epsilon, l}+\mathbf{N}_{l}
$$

where $\mathbf{H}_{l}$ and $\mathbf{N}_{l}$ denote the channel state and noise matrices, respectively. The quantity $\mathbf{X}_{\epsilon, l}$ denotes the $N_{t} \times N_{c}$ matrix of symbols $x_{i}^{\epsilon}[n]$. Using (6), we express $\mathbf{X}_{\epsilon, l}$ as

$$
\mathbf{X}_{\epsilon, l}=\sum_{n} \mathbf{X}_{l+n} \mathbf{G}_{\epsilon, n}
$$

where $\mathbf{G}_{\epsilon, n}$ is a $N_{c} \times N_{c}$ Toeplitz matrix given by

$$
\mathbf{G}_{\epsilon, n}=\left[\begin{array}{cccc}
g_{-n N_{c}}^{\epsilon} & g_{-n N_{c}+1}^{\epsilon} & g_{-n N_{c}+2}^{\epsilon} & \cdots \\
g_{-n N_{c}-1}^{\epsilon} & g_{-n N_{c}}^{\epsilon} & g_{-n N_{c}+1}^{\epsilon} & \ddots \\
g_{-n N_{c}-2}^{\epsilon} & g_{-n N_{c}-1}^{\epsilon} & g_{-n N_{c}}^{\epsilon} & \ddots \\
\vdots & \ddots & \ddots & \ddots
\end{array}\right]
$$

where we denote the pulse shape samples by $g_{n}^{\epsilon} \triangleq g(n T+\epsilon)$.

Finally, the detection variables for each information symbol $m=0, \ldots, N_{s}-1$ within block $l$ are given by [10]

$$
s_{m, l}=\left\|\mathbf{H}_{l}\right\|^{-2}\left[\Re\left\{\operatorname{tr}\left(\mathbf{Y}_{l}^{H} \mathbf{H}_{l} \mathbf{A}_{m}\right)\right\}-i \Im\left\{\operatorname{tr}\left(\mathbf{Y}_{l}^{H} \mathbf{H}_{l} \mathbf{B}_{m}\right)\right\}\right]
$$

where $\mathbf{A}_{m}$ and $\mathbf{B}_{m}$ are the encoding matrices used in (1), $\operatorname{tr}(\cdot)$ denotes the trace operator, superscript $H$ is the Hermitian transpose operator and $\left\|\mathbf{H}_{l}\right\|$ is the Frobenius norm of $\mathbf{H}_{l}$. We note that strictly speaking (10) represents ML detection when no timing error is present. However, for small values of $\epsilon$ considered here, we assume that (10) is an close approximation to ML detection. The projection of $s_{m, l}$ onto the signal constellation then forms the data decisions denoted by $\hat{a}_{m, l}$. 


\section{TIMING ERRor Detector DESIGN}

It was shown in [8] that for a 2-transmit antenna OSTBC, a TED in the form of $\hat{\epsilon}=\Re\left\{a_{0} s_{1}-a_{1} s_{0}\right\}$ results in an Scurve, that is the expectation $E\{\hat{\epsilon}\}$, of $E\{\hat{\epsilon}\}=g_{-1}^{\epsilon}-g_{1}^{\epsilon}$. The expression $g_{-1}^{\epsilon}-g_{1}^{\epsilon}$ has been referred to in literature [7] as the difference-of-threshold-crossings. We refer to $C\left(g_{-1}^{\epsilon}-g_{1}^{\epsilon}\right)$, where $C$ is a constant, as the TEM. Under the assumption of perfect channel knowledge at the receiver the above TEM is independent of the channel state, giving robustness in poor channel conditions. We will define a TED whose TEM is independent of the channel fading process as a robust TED. It will be shown that $E\{\hat{\epsilon}\}$ for higher order OSTBC is composed of a dominant TEM term and a bias which is a rational function of quadratic forms with a denominator containing magnitude terms of the channel states, and a numerator consisting only of cross products of the channel variables. Under the realistic condition of slow timing drift, the average of the TED bias taken over the channel fading, which in practice is carried out by virtue of the iterative operation of the timing loop, will thus be small. Such TEDs will be referred to as quasi-robust.

In what follows, we derive sufficient conditions for the design of robust or quasi-robust timing error detectors. We point out that the following conditions are not necessary for a valid TED - other methods may also lead to TEM functions.

\section{A. Conditions for TED Robustness}

We consider a general expression for an estimate of $\epsilon$ in the form of a linear combination of products ${ }^{3} a_{n} s_{m}$ and $a_{n}^{*} s_{m}$, that is

$$
\hat{\epsilon}=\Re\left(\sum_{k} \alpha_{k} a_{n_{\alpha, k}} s_{m_{\alpha, k}}+\beta_{k} a_{n_{\beta, k}}^{*} s_{m_{\beta, k}}\right)
$$

where $n_{\alpha, k}, m_{\alpha, k}, n_{\beta, k}$ and $m_{\beta, k}$ denote the data and decision metric indices chosen for each TED term corresponding to $\alpha_{k}$ and $\beta_{k}$. From (11) we define a set

$$
\mathcal{S}=\left\{\alpha_{k}, \beta_{k}, m_{\alpha, k}, n_{\alpha, k}, m_{\beta, k}, n_{\beta, k}\right\}
$$

which contains sum weights as well as the indices within a block of data symbols and decision variables to be chosen. In (11) we have ignored the imaginary component of the estimator. The design problem is to choose the parameters in the set $\mathcal{S}$ to obtain a TEM that is close to $C\left(g_{-1}^{\epsilon}-g_{1}^{\epsilon}\right)$.

We examine the expectation $E\{\hat{\epsilon}\}$, taken over the data, noise and the channel state. Assuming that the channel fading is independent of data and channel noise, we will first evaluate the expectation over the information symbols and the noise, conditioned on the channel matrix $\mathbf{H}$. We will denote such an operator by ${ }^{4} E^{\mathbf{H}}\{\hat{\epsilon}\}$. The complete expectation $E\{\hat{\epsilon}\}$ can then be obtained by evaluating the expectation of $E^{\mathbf{H}}\{\hat{\epsilon}\}$ over the channel matrix $\mathbf{H}$, that is

$$
E\{\hat{\epsilon}\}=E_{\mathbf{H}}\left\{E^{\mathbf{H}}\{\hat{\epsilon}\}\right\}
$$

\footnotetext{
${ }^{3}$ In the interest of clarity, we remove the block index $l$ from the notation, with the dependence of $a_{n}, s_{m}, \epsilon$ and $\hat{\epsilon}$ on $l$ implied.

${ }^{4}$ A compact notation of $E^{\mathbf{H}}\{\cdot\}$ is chosen over the standard $E\{\cdot \mid \mathbf{H}\}$, in an attempt to simplify expressions in which the arguments of the expectation operator are very involved.
}

where $E_{\mathbf{H}}\{\cdot\}$ is the expectation with respect to $\mathbf{H}$, which, as will be shown, must be carried out via simulation. In evaluating $E^{\mathbf{H}}\{\hat{\epsilon}\}$ we consider the individual components of the summation in (11), specifically $E^{\mathbf{H}}\left\{a_{n} s_{m}\right\}$ and $E^{\mathbf{H}}\left\{a_{n}^{*} s_{m}\right\}$. The derivation is presented in Appendix A, where it is shown that

$$
\begin{aligned}
& E^{\mathbf{H}}\left\{a_{n} s_{m}\right\}=\rho_{2}\|\mathbf{H}\|^{-2} \times \\
& \quad\left[\operatorname{tr}\left\{\left(\mathbf{A}_{m} \mathbf{G}_{\epsilon}^{H} \mathbf{A}_{n}^{H}-\mathbf{B}_{m} \mathbf{G}_{\epsilon}^{H} \mathbf{B}_{n}^{H}\right) \Re\left(\mathbf{H}^{H} \mathbf{H}\right)\right\}\right. \\
& \left.-j \operatorname{tr}\left\{\left(\mathbf{A}_{m} \mathbf{G}_{\epsilon}^{H} \mathbf{B}_{n}^{H}-\mathbf{B}_{m} \mathbf{G}_{\epsilon}^{H} \mathbf{A}_{n}^{H}\right) \Im\left(\mathbf{H}^{H} \mathbf{H}\right)\right\}\right]
\end{aligned}
$$

and

$$
\begin{aligned}
& E^{\mathbf{H}}\left\{a_{n}^{*} s_{m}\right\}=\rho_{2}\|\mathbf{H}\|^{-2} \times \\
& \quad\left[\operatorname{tr}\left\{\left(\mathbf{A}_{m} \mathbf{G}_{\epsilon}^{H} \mathbf{A}_{n}^{H}+\mathbf{B}_{m} \mathbf{G}_{\epsilon}^{H} \mathbf{B}_{n}^{H}\right) \Re\left(\mathbf{H}^{H} \mathbf{H}\right)\right\}\right. \\
& \left.-j \operatorname{tr}\left\{\left(\mathbf{A}_{m} \mathbf{G}_{\epsilon}^{H} \mathbf{B}_{n}^{H}+\mathbf{B}_{m} \mathbf{G}_{\epsilon}^{H} \mathbf{A}_{n}^{H}\right) \Im\left(\mathbf{H}^{H} \mathbf{H}\right)\right\}\right]
\end{aligned}
$$

where $\mathbf{A}_{n}, \mathbf{B}_{n}$ are the code matrices in (1), and $\mathbf{G}_{\epsilon}$ represents $\mathbf{G}_{\epsilon, n}$ for $n=0$, as defined by (9). We have used superscripts $R$ and $I$ to denote real and imaginary components and have defined a constellation-dependent constant

$$
\rho_{p} \triangleq E\left\{\left(a_{i}^{R}\right)^{p}\right\}=E\left\{\left(a_{i}^{I}\right)^{p}\right\} .
$$

Using (14) and (15), one obtains the expectation of the TED in (11) given by

$$
E^{\mathbf{H}}\{\hat{\epsilon}\}=\rho_{2}\|\mathbf{H}\|^{-2} \operatorname{tr}\left\{\boldsymbol{\Gamma} \Re\left(\mathbf{H}^{H} \mathbf{H}\right)\right\}
$$

where we have defined a matrix $\boldsymbol{\Gamma}$, dependent on the coefficient set $\mathcal{S}$ chosen in (12), as

$$
\begin{aligned}
& \boldsymbol{\Gamma}=\sum_{k} {\left[\alpha_{k}\left(\mathbf{A}_{m_{\alpha, k}} \mathbf{G}_{\epsilon}^{H} \mathbf{A}_{n_{\alpha, k}}^{H}-\mathbf{B}_{m_{\alpha, k}} \mathbf{G}_{\epsilon}^{H} \mathbf{B}_{n_{\alpha, k}}^{H}\right)\right.} \\
&\left.+\beta_{k}\left(\mathbf{A}_{m_{\beta, k}} \mathbf{G}_{\epsilon}^{H} \mathbf{A}_{n_{\beta, k}}^{H}+\mathbf{B}_{m_{\beta, k}} \mathbf{G}_{\epsilon}^{H} \mathbf{B}_{n_{\beta, k}}^{H}\right)\right] .
\end{aligned}
$$

The design problem now reduces to a consideration of the matrix $\Gamma$ in (18). Consider the case where $\Gamma$ in (18) has the form of

$$
\boldsymbol{\Gamma}=f\left(\mathbf{G}_{\epsilon}\right) \mathbf{I}+\mathbf{D}
$$

where

1) $f\left(\mathbf{G}_{\epsilon}\right)$ is a scalar function of $\mathbf{G}_{\epsilon}$ that returns a TEM, usually $g_{-1}^{\epsilon}-g_{1}^{\epsilon}$

2) $\mathbf{D}$ is an antisymmetric matrix.

Then, using (17)

$$
\begin{aligned}
E^{\mathbf{H}}\{\hat{\epsilon}\} & =\rho_{2}\|\mathbf{H}\|^{-2} \operatorname{tr}\left\{\left(f\left(\mathbf{G}_{\epsilon}\right) \mathbf{I}+\mathbf{D}\right) \Re\left(\mathbf{H}^{H} \mathbf{H}\right)\right\} \\
& =\rho_{2} f\left(\mathbf{G}_{\epsilon}\right) \\
& =E\{\hat{\epsilon}\}
\end{aligned}
$$

where deriving (20) we have used the following properties

- $\Re\left(\mathbf{H}^{H} \mathbf{H}\right)$ is symmetric

- $\operatorname{tr}\{\mathbf{A B}\}=0$ for symmetric $\mathbf{A}$ and antisymmetric $\mathbf{B}$

- $\operatorname{tr}\left\{\Re\left(\mathbf{H}^{H} \mathbf{H}\right)\right\}=\|\mathbf{H}\|^{2}$.

Therefore, if coefficients in (11) are selected such that $\Gamma$ satisfies conditions 1 ) and 2), the TED returns a valid timing error measurement that is robust to channel fading 5 . As

\footnotetext{
${ }^{5}$ In other words, the $\operatorname{tr}\{\cdot\}$ operator in (20) returns a full-diversity measurement of $\epsilon$, which removes any dependence on $\mathbf{H}$.
} 
indicated by (20), no averaging over $\mathbf{H}$ is required to obtain the full expectation of $\hat{\epsilon}$.

If only condition 1 is satisfied, that is $\mathbf{D}$ is an arbitrary matrix with zeros on the main diagonal, then

$$
E^{\mathbf{H}}\{\hat{\epsilon}\}=\mu+\delta_{\hat{\epsilon}}
$$

where we have defined $\mu=\rho_{2} f\left(\mathbf{G}_{\epsilon}\right)$ as the desired TEM signal and $\delta_{\hat{\epsilon}}$, dependent on $\mathbf{H}$, as the TEM bias. The S-curve, that is the expectation of $\hat{\epsilon}$ over all random variables, is thus obtained from (21) by

$$
E\{\hat{\epsilon}\}=\mu+\bar{\delta}_{\hat{\epsilon}}
$$

where the average bias $\bar{\delta}_{\hat{\epsilon}}$ is given by

$$
\bar{\delta}_{\hat{\epsilon}} \triangleq E_{\mathbf{H}}\left\{\delta_{\hat{\epsilon}}\right\} .
$$

One can show using (20) that

$$
\delta_{\hat{\epsilon}}=\rho_{2}\|\mathbf{H}\|^{-2} \sum_{m=1}^{N_{t}} \sum_{\substack{i=1 \\ i \neq m}}^{N_{t}} \sum_{j=1}^{N_{r}} d_{m i} \Re\left(h_{j i}^{*} h_{j m}\right)
$$

where we used $d_{m i}$ to denote the $(m, i)$ th entry of $\mathbf{D}$. We note that the denominator of the bias contains magnitude terms of the channel states, while the numerator is made up of cross products of the channel variables. Thus, for uncorrelated channels, the expectation of the bias taken over $\mathbf{H}$ will be relatively small, resulting in a quasi-robust TED. Equation (24) also confirms that if the antisymmetry condition for $\mathbf{D}$ is satisfied, that is, $d_{m i}=-d_{i m}$, then $\delta_{\hat{\epsilon}}=0$ giving zero bias and a robust TEM.

Recall that the desired design output is one satisfying conditions 1) and 2) above (20). Examining (18), we see that the integer code matrices $\mathbf{A}_{m}$ and $\mathbf{B}_{m}$ act to shuffle the rows and columns of $\mathbf{G}_{\epsilon}^{H}$. Thus the design step is to force the elements $g_{-1}^{\epsilon}$ and $g_{1}^{\epsilon}$, located adjacent to the main diagonal of $\mathbf{G}_{\epsilon}$, to the main diagonal of $\boldsymbol{\Gamma}$ for $k=1$ and $k=2$, through the choosing of $\mathcal{S}$ in (12). Since the composition of the code matrices $\mathbf{A}_{m}$ and $\mathbf{B}_{m}$ in (1) varies for different OSTBCs, the procedure must be repeated for each code under consideration. We note that the resulting design may not be unique. Furthermore, while in [11] it was shown that the design method presented herein produced valid TEMs for all codes considered, no proof of existence of a valid solution to $\mathcal{S}$ for every OSTBC has been found. Note that while the design assumes perfect receiver channel knowledge, the performance analysis via simulation presented in Section V considers the effects of channel estimation and data decision errors. Design criteria for unknown channels are a worthwhile extension to the work presented here.

\section{B. TED Variance and Output SNR}

In this section we derive the variance of the timing error estimate produced by the TEDs described in Section III-A. The solution, together with the expression for the S-curve in (17) allows us to obtain the output SNR of the detector.

The variance of the TED output is defined by

$$
\sigma_{\hat{\epsilon}}^{2}=E\left\{\hat{\epsilon}^{2}\right\}-[E\{\hat{\epsilon}\}]^{2} .
$$

Similarly to the method in Section III-A, in evaluating the expectation $E\left\{\hat{\epsilon}^{2}\right\}$ we first consider the expectation over the data and noise, conditioned on the channel state $\mathbf{H}$. Once again, the expectation over $\mathbf{H}$ must be computed using a numerical approach.

By examining (11), one can see that the solution to $E^{\mathbf{H}}\left\{\hat{\epsilon}^{2}\right\}$ for various TED formulations can be obtained by considering $E^{\mathbf{H}}\left\{a_{i}^{R} a_{j}^{R} s_{m}^{R} s_{n}^{R}\right\}, E^{\mathbf{H}}\left\{a_{i}^{I} a_{j}^{I} s_{m}^{I} s_{n}^{I}\right\}$ and $E^{\mathbf{H}}\left\{a_{i}^{R} a_{j}^{I} s_{m}^{R} s_{n}^{I}\right\}$. We begin with $E^{\mathbf{H}}\left\{a_{i}^{R} a_{j}^{R} s_{m}^{R} s_{n}^{R}\right\}$, which from (10) can be expressed as

$$
\begin{aligned}
E^{\mathbf{H}}\left\{a_{i}^{R} a_{j}^{R} s_{m}^{R} s_{n}^{R}\right\}=\|\mathbf{H}\|^{-4} E^{\mathbf{H}}\left\{a_{i}^{R} a_{j}^{R} \times\right. \\
\left.\operatorname{tr}\left\{\mathbf{A}_{m} \Re\left(\mathbf{Y}^{H} \mathbf{H}\right)\right\} \operatorname{tr}\left\{\mathbf{A}_{n} \Re\left(\mathbf{Y}^{H} \mathbf{H}\right)\right\}\right\}
\end{aligned}
$$

where we used the fact that $\Re(\operatorname{tr}(\cdot))=\operatorname{tr}(\Re(\cdot)), \operatorname{tr}(\mathbf{A B})=$ $\operatorname{tr}(\mathbf{B A})$ and that the matrices $\mathbf{A}_{m}$ and $\mathbf{A}_{n}$ are real valued. Assuming that the data is drawn from a symmetrical constellation, and is independent from the noise, the solution to (26), details of which are presented in Appendix B, is given by

$$
E^{\mathbf{H}}\left\{a_{i}^{R} a_{j}^{R} s_{m}^{R} s_{n}^{R}\right\}=\|\mathbf{H}\|^{-4} \operatorname{tr}\left\{\rho_{2}^{2} \boldsymbol{\Phi}_{i j m n}^{R R}+\rho_{2} \frac{N_{0}}{2} \boldsymbol{\Delta}_{i j m n}^{R R}\right\}
$$

where $\boldsymbol{\Phi}_{i j m n}^{R R}$ is defined by (28) and

$$
\boldsymbol{\Delta}_{i j m n}^{R R}= \begin{cases}0 & i \neq j \\ \left(\mathbf{A}_{m} \otimes \mathbf{A}_{n}\right) \boldsymbol{\Lambda}_{N}\left(\boldsymbol{\Omega}_{\mathrm{RR}}^{\prime}+\boldsymbol{\Omega}_{\mathrm{II}}^{\prime}\right) & i=j\end{cases}
$$

with $\otimes$ denoting the Kronecker matrix product. In (28), $\rho_{p}$ is defined by (16), whereas the constant $\rho_{p}^{\prime}$ is given by

$$
\rho_{p}^{\prime} \triangleq E\left\{\left(a_{i}^{R}\right)^{p}\left(a_{i}^{I}\right)^{p}\right\} .
$$

The $N_{c} N_{c} \times N_{r} N_{r}$ matrix $\boldsymbol{\Lambda}_{N}$ in (29) is given by

$$
\boldsymbol{\Lambda}_{N}(i, j)= \begin{cases}1 & i=n N_{r}+n+1, j=m N_{c}+m+1 \\ 0 & \text { otherwise }\end{cases}
$$

for $n=0, \ldots, N_{r}-1$ and $m=0, \ldots, N_{c}-1$. In (28) and (29) we have defined channel dependent variables

$$
\begin{aligned}
\boldsymbol{\Omega}_{\mathrm{RR}} & =\Re\left(\mathbf{H}^{H} \mathbf{H}\right) \otimes \Re\left(\mathbf{H}^{H} \mathbf{H}\right) \\
\boldsymbol{\Omega}_{\mathrm{II}} & =\Im\left(\mathbf{H}^{H} \mathbf{H}\right) \otimes \Im\left(\mathbf{H}^{H} \mathbf{H}\right) \\
\boldsymbol{\Omega}_{\mathrm{RI}} & =\Re\left(\mathbf{H}^{H} \mathbf{H}\right) \otimes \Im\left(\mathbf{H}^{H} \mathbf{H}\right) \\
\boldsymbol{\Omega}_{\mathrm{IR}} & =\Im\left(\mathbf{H}^{H} \mathbf{H}\right) \otimes \Re\left(\mathbf{H}^{H} \mathbf{H}\right)
\end{aligned}
$$

and

$$
\begin{aligned}
\boldsymbol{\Omega}_{\mathrm{RR}}^{\prime} & =\Re(\mathbf{H}) \otimes \Re(\mathbf{H}) \\
\boldsymbol{\Omega}_{\mathrm{II}}^{\prime} & =\Im(\mathbf{H}) \otimes \Im(\mathbf{H}) \\
\boldsymbol{\Omega}_{\mathrm{RI}}^{\prime} & =\Re(\mathbf{H}) \otimes \Im(\mathbf{H}) \\
\boldsymbol{\Omega}_{\mathrm{IR}}^{\prime} & =\Im(\mathbf{H}) \otimes \Re(\mathbf{H}) .
\end{aligned}
$$

The solution to $E^{\mathbf{H}}\left\{a_{i}^{I} a_{j}^{I} s_{m}^{I} s_{n}^{I}\right\}$ can be obtained by following the same methodology as for $E^{\mathbf{H}}\left\{a_{i}^{R} a_{j}^{R} s_{m}^{R} s_{n}^{R}\right\}$. The details are once again shown in Appendix B, with the solution given by

$$
E^{\mathbf{H}}\left\{a_{i}^{I} a_{j}^{I} s_{m}^{I} s_{n}^{I}\right\}=\|\mathbf{H}\|^{-4} \operatorname{tr}\left\{\rho_{2}^{2} \boldsymbol{\Phi}_{i j m n}^{I I}+\rho_{2} \frac{N_{0}}{2} \boldsymbol{\Delta}_{i j m n}^{I I}\right\}
$$




$$
\begin{aligned}
\mathbf{\Phi}_{i j m n}^{R R}= \begin{cases}\left(\mathbf{A}_{m} \mathbf{G}_{\epsilon, 0}^{H} \otimes \mathbf{A}_{n} \mathbf{G}_{\epsilon, 0}^{H}\right)\left(\mathbf{A}_{j}^{H} \otimes \mathbf{A}_{i}^{H}+\mathbf{A}_{i}^{H} \otimes \mathbf{A}_{j}^{H}\right) \boldsymbol{\Omega}_{R R} & i \neq j \\
\left(\mathbf{A}_{m} \mathbf{G}_{\epsilon, 0}^{H} \otimes \mathbf{A}_{n} \mathbf{G}_{\epsilon, 0}^{H}\right)\left[\left(\frac{\rho_{4}}{\rho_{2}^{2}}-1\right)\left(\mathbf{A}_{i}^{H} \otimes \mathbf{A}_{i}^{H}\right) \boldsymbol{\Omega}_{R R}+\left(\frac{\rho_{2}^{\prime}}{\rho_{2}}-1\right)\left(\mathbf{B}_{i}^{H} \otimes \mathbf{B}_{i}^{H}\right) \boldsymbol{\Omega}_{\mathrm{II}}\right] & \\
+\sum_{l} \sum_{k=0}^{N_{s}-1}\left(\mathbf{A}_{m} \mathbf{G}_{\epsilon, l}^{H} \otimes \mathbf{A}_{n} \mathbf{G}_{\epsilon, l}^{H}\right)\left[\left(\mathbf{A}_{k}^{H} \otimes \mathbf{A}_{k}^{H}\right) \boldsymbol{\Omega}_{R R}+\left(\mathbf{B}_{k}^{H} \otimes \mathbf{B}_{k}^{H}\right) \boldsymbol{\Omega}_{\mathrm{II}}\right] & i=j\end{cases} \\
\mathbf{\Phi}_{i j m n}^{I I}= \begin{cases}\left(\mathbf{B}_{m} \mathbf{G}_{\epsilon, 0}^{H} \otimes \mathbf{B}_{n} \mathbf{G}_{\epsilon, 0}^{H}\right)\left(\mathbf{B}_{j}^{H} \otimes \mathbf{B}_{i}^{H}+\mathbf{B}_{i}^{H} \otimes \mathbf{B}_{j}^{H}\right) \boldsymbol{\Omega}_{R R} & i \neq j \\
\left(\mathbf{B}_{m} \mathbf{G}_{\epsilon, 0}^{H} \otimes \mathbf{B}_{n} \mathbf{G}_{\epsilon, 0}^{H}\right)\left[\left(\frac{\rho_{4}}{\rho_{2}^{2}}-1\right)\left(\mathbf{B}_{i}^{H} \otimes \mathbf{B}_{i}^{H}\right) \boldsymbol{\Omega}_{R R}+\left(\frac{\rho_{2}^{\prime}}{\rho_{2}^{2}}-1\right)\left(\mathbf{A}_{i}^{H} \otimes \mathbf{A}_{i}^{H}\right) \boldsymbol{\Omega}_{\mathrm{II}}\right] & \\
+\sum_{l} \sum_{k=0}^{N_{s}-1}\left(\mathbf{B}_{m} \mathbf{G}_{\epsilon, l}^{H} \otimes \mathbf{B}_{n} \mathbf{G}_{\epsilon, l}^{H}\right)\left[\left(\mathbf{B}_{k}^{H} \otimes \mathbf{B}_{k}^{H}\right) \boldsymbol{\Omega}_{R R}+\left(\mathbf{A}_{k}^{H} \otimes \mathbf{A}_{k}^{H}\right) \boldsymbol{\Omega}_{\mathrm{II}}\right] & i=j\end{cases}
\end{aligned}
$$

with $\boldsymbol{\Phi}_{i j m n}^{I I}$ is given by (35) and

$$
\boldsymbol{\Delta}_{i j m n}^{I I}= \begin{cases}0 & i \neq j \\ \left(\mathbf{B}_{m} \otimes \mathbf{B}_{n}\right) \boldsymbol{\Lambda}_{N}\left(\boldsymbol{\Omega}_{\mathrm{RR}}^{\prime}+\boldsymbol{\Omega}_{\mathrm{II}}^{\prime}\right) & i=j .\end{cases}
$$

Finally, the expectation $E^{\mathbf{H}}\left\{a_{i}^{R} a_{j}^{I} s_{m}^{R} s_{n}^{I}\right\}$, as outlined in Appendix $\mathrm{B}$, is given by

$$
E^{\mathbf{H}}\left\{a_{i}^{R} a_{j}^{I} s_{m}^{R} s_{n}^{I}\right\}=\|\mathbf{H}\|^{-4} \operatorname{tr}\left\{\tilde{\rho} \boldsymbol{\Phi}_{i j m n}^{R I}\right\}
$$

where

$$
\begin{aligned}
\boldsymbol{\Phi}_{i j m n}^{R I}= & \left(\mathbf{A}_{m} \mathbf{G}_{\epsilon, 0}^{H} \otimes \mathbf{B}_{n} \mathbf{G}_{\epsilon, 0}^{H}\right) \times \\
& \left(\left(\mathbf{A}_{i}^{H} \otimes \mathbf{B}_{j}^{H}\right) \boldsymbol{\Omega}_{\mathrm{RR}}-\left(\mathbf{B}_{j}^{H} \otimes \mathbf{A}_{i}^{H}\right) \boldsymbol{\Omega}_{\mathrm{II}}\right)
\end{aligned}
$$

and $\tilde{\rho}$ is defined as

$$
\tilde{\rho}= \begin{cases}\rho_{2}^{2} & i \neq j \\ \rho_{2}^{\prime} & i=j .\end{cases}
$$

Using (27), (34) and (37), one can obtain $E\left\{\hat{\epsilon}^{2}\right\}$ for a particular TED, that is

$$
E\left\{\hat{\epsilon}^{2}\right\}=E_{\mathbf{H}}\left\{\|\mathbf{H}\|^{-4} \operatorname{tr}\left\{\rho_{2}^{2} \boldsymbol{\Sigma}_{\boldsymbol{\Phi}}+\rho_{2} \frac{N_{0}}{2} \boldsymbol{\Sigma}_{\boldsymbol{\Delta}}\right\}\right\}
$$

where $\boldsymbol{\Sigma}_{\boldsymbol{\Phi}}$ and $\boldsymbol{\Sigma}_{\boldsymbol{\Delta}}$ correspond to the linear combinations of $\boldsymbol{\Phi}_{i j m n}^{R R}, \boldsymbol{\Phi}_{i j m n}^{I I}, \boldsymbol{\Phi}_{i j m n}^{R I}$ and $\boldsymbol{\Delta}_{i j m n}^{R R}, \boldsymbol{\Delta}_{i j m n}^{I I}$, respectively, as determined by the polynomial expansion of $E\left\{\hat{\epsilon}^{2}\right\}$ for a particular TED. Expressions for specific TEDs will be derived in Section III-C.

Finally, we can define the TED SNR as

$$
\begin{aligned}
S N R_{T E D} & =\frac{\mu^{2}}{E\left\{(\hat{\epsilon}-\mu)^{2}\right\}} \\
& =\frac{\mu^{2}}{\sigma_{\hat{\epsilon}}^{2}+\bar{\delta}_{\hat{\epsilon}}^{2}}
\end{aligned}
$$

where the numerator represents the power of the TEM signal and the denominator is the equivalent noise power, with $\sigma_{\hat{\epsilon}}^{2}$ defined by (25) with (40) and $\bar{\delta}_{\hat{\epsilon}}$ given by (23). As discussed in Section III-A, the effect of the TEM bias is small, and thus one can approximate (41) by

$$
S N R_{T E D} \approx \frac{[E\{\hat{\epsilon}\}]^{2}}{\sigma_{\hat{\epsilon}}^{2}} .
$$

\section{Examples of TEDs}

Based on the design conditions for satisfying TED robustness described in Section III-A, we now derive examples of TEDs for a number of specific OSTBC codes, obtaining expressions for the S-curve and the estimation variance ${ }^{6}$. We note that the examples given here do not represent unique solutions the parameter set $\mathcal{S}$ in (12). Other instances of $\mathcal{S}$ may lead to similar TEM functions.

As proposed in [8], a TED for a 2-transmit antenna OSTBC (Alamouti encoding) has the form of

$$
\begin{aligned}
\hat{\epsilon}_{(2)} & =\Re\left\{a_{0} s_{1}-a_{1} s_{0}\right\} \\
& =a_{1}^{R} s_{0}^{R}-a_{1}^{I} s_{0}^{I}-a_{0}^{R} s_{1}^{R}+a_{0}^{I} s_{1}^{I}
\end{aligned}
$$

which corresponds to $\alpha_{1}=1, \alpha_{2}=-1, m_{\alpha, 1}=n_{\alpha, 2}=1$, $m_{\alpha, 2}=n_{\alpha, 1}=0$ and $\beta_{k}=0$ in the set $\mathcal{S}$ in (12) for all $k$. For this case, the matrix $\Gamma$ in (18) can be shown to be

$$
\boldsymbol{\Gamma}_{(2)}=2\left[\begin{array}{cc}
g_{-1}^{\epsilon}-g_{1}^{\epsilon} & 0 \\
0 & g_{-1}^{\epsilon}-g_{1}^{\epsilon}
\end{array}\right]
$$

that is, $f\left(\mathbf{G}_{\epsilon}\right)=2\left(g_{-1}^{\epsilon}-g_{1}^{\epsilon}\right)$ and $\mathbf{D}=0$, thus satisfying design conditions 1) and 2) described in Section III-A. Consistent with the results in [8], the S-curve of the TED in (43) is given by $E\left\{\hat{\epsilon}_{(2)}\right\}=\mu=2 \rho_{2}\left(g_{-1}^{\epsilon}-g_{1}^{\epsilon}\right)$, that is a robust timing estimate with $\delta_{\hat{\epsilon}_{(2)}}=0$.

The TED SNR for $\hat{\epsilon}_{(2)}$ can be calculated using (41) (with $\bar{\delta}_{\hat{\epsilon}}=0$ ), where there the equivalent noise power is computed using (25) and (40), with

$$
\begin{aligned}
\boldsymbol{\Sigma}_{\boldsymbol{\Phi}} & =\boldsymbol{\Phi}_{1100}^{R R}+\boldsymbol{\Phi}_{0011}^{R R}-2 \boldsymbol{\Phi}_{1010}^{R R}+\boldsymbol{\Phi}_{1100}^{I I}+\boldsymbol{\Phi}_{0011}^{I I} \\
& -2 \boldsymbol{\Phi}_{1010}^{I I}-2 \boldsymbol{\Phi}_{1100}^{R I}-2 \boldsymbol{\Phi}_{0011}^{R I}+2 \boldsymbol{\Phi}_{1001}^{R I}+2 \boldsymbol{\Phi}_{0110}^{R I}
\end{aligned}
$$

and

$$
\boldsymbol{\Sigma}_{\boldsymbol{\Delta}}=\boldsymbol{\Delta}_{1100}^{R R}+\boldsymbol{\Delta}_{0011}^{R R}+\boldsymbol{\Delta}_{1100}^{I I}+\boldsymbol{\Delta}_{0011}^{I I} .
$$

A number of $N_{t}=3$ OSTBC encoders have been presented in literature, such as [14, Equations 7.4.8, 7.4.9] and [15, Equation 3.49], all of rate $R=3 / 4$. While similar in structure, each of these codes will require a separately designed TEDs. Consider the code in Equation 3.49 of [15], denoted by

$$
\mathbf{X}_{(3)}=\left[\begin{array}{cccc}
a_{0} & a_{1}^{*} & a_{2}^{*} & 0 \\
-a_{1} & a_{0}^{*} & 0 & -a_{2}^{*} \\
-a_{2} & 0 & a_{0}^{*} & a_{1}^{*}
\end{array}\right]
$$

${ }^{6}$ For more examples of TED expressions, including those for rate-one realvalued OSTBCs, the reader is referred to [11]. 
Referring to (18), one can show that, for $k=1$, selecting $\alpha_{1}=\beta_{1}=1$ with $n_{\alpha, 1}=n_{\beta, 1}=1$ and $m_{\alpha, 1}=m_{\beta, 1}=0$ in $\mathcal{S}$ will cause matrices $\mathbf{A}_{0}, \mathbf{B}_{0}, \mathbf{A}_{1}^{H}$ and $\mathbf{B}_{1}^{H}$ to force the main diagonal of $\boldsymbol{\Gamma}$ to be $\left\{2 g_{-1}^{\epsilon},-2 g_{1}^{\epsilon}, 2 g_{-1}^{\epsilon}\right\}$. Similarly, for $k=2$, choosing $\alpha_{2}=\beta_{2}=-1$ with $n_{\alpha, 2}=n_{\beta, 2}=0$ and $m_{\alpha, 2}=$ $m_{\beta, 2}=1$, contributes $\left\{-2 g_{1}^{\epsilon}, 2 g_{-1}^{\epsilon},-2 g_{1}^{\epsilon}\right\}$. Subtracting the $k=2$ term from the $k=1$ term, which is equivalent to a TED in the form of

$$
\begin{aligned}
\hat{\epsilon}_{(3)} & =\Re\left\{a_{1} s_{0}-a_{0} s_{1}+a_{1}^{*} s_{0}-a_{0}^{*} s_{1}\right\} \\
& =2\left(a_{1}^{R} s_{0}^{R}-a_{0}^{R} s_{1}^{R}\right)
\end{aligned}
$$

results in $\boldsymbol{\Gamma}_{(3)}$ given by

$$
\boldsymbol{\Gamma}_{(3)}=2\left[\begin{array}{ccc}
g_{-1}^{\epsilon}-g_{1}^{\epsilon} & -2 g_{0}^{\epsilon} & g_{-3}^{\epsilon}-g_{-1}^{\epsilon} \\
2 g_{0}^{\epsilon} & g_{-1}^{\epsilon}-g_{1}^{\epsilon} & 2 g_{-2}^{\epsilon} \\
g_{1}^{\epsilon}-g_{3}^{\epsilon} & -2 g_{2}^{\epsilon} & g_{-1}^{\epsilon}-g_{1}^{\epsilon}
\end{array}\right]
$$

that is $f\left(\mathbf{G}_{\epsilon}\right)=2\left(g_{-1}^{\epsilon}-g_{1}^{\epsilon}\right)$ and

$$
\mathbf{D}_{(3)}=2\left[\begin{array}{ccc}
0 & -2 g_{0}^{\epsilon} & g_{-3}^{\epsilon}-g_{-1}^{\epsilon} \\
2 g_{0}^{\epsilon} & 0 & 2 g_{-2}^{\epsilon} \\
g_{1}^{\epsilon}-g_{3}^{\epsilon} & -2 g_{2}^{\epsilon} & 0
\end{array}\right] .
$$

By examining (49) and (50), we note that the formulation of (48) satisfies design condition 1), resulting in a quasi-robust TED. The S-curve for the TED in (48) is given by

$$
E\left\{\hat{\epsilon}_{(3)}\right\}=2 \rho_{2}\left(g_{-1}^{\epsilon}-g_{1}^{\epsilon}\right)+E_{\mathbf{H}}\left\{\delta_{\hat{\epsilon}_{(3)}}\right\}
$$

that is a TEM component of $\mu=2 \rho_{2}\left(g_{-1}^{\epsilon}-g_{1}^{\epsilon}\right)$ and a bias term where

$$
\begin{aligned}
& \delta_{\hat{\epsilon}_{(3)}}=\|\mathbf{H}\|^{-2} 2 \rho_{2} \sum_{j=1}^{N_{r}}\left[2\left(g_{-2}^{\epsilon}-g_{2}^{\epsilon}\right) \Re\left(h_{j 3}^{*} h_{j 2}\right)\right. \\
& \left.-\left(g_{-1}^{\epsilon}-g_{1}^{\epsilon}-g_{-3}^{\epsilon}+g_{3}^{\epsilon}\right) \Re\left(h_{j 3}^{*} h_{j 1}\right)\right] .
\end{aligned}
$$

As discussed earlier, the numerator in (52) contains only cross product terms of the channel coefficients, while $\|\mathbf{H}\|$ contains magnitude terms. The bias in (52) will be small relative to first term in (51), and thus the TED is quasi-robust.

The TED SNR can be solved using (41), with the equivalent noise power computed using (25) and (40), with

$$
\boldsymbol{\Sigma}_{\boldsymbol{\Phi}}=4\left(\boldsymbol{\Phi}_{1100}^{R R}+\boldsymbol{\Phi}_{0011}^{R R}-2 \boldsymbol{\Phi}_{1010}^{R R}\right)
$$

and

$$
\boldsymbol{\Sigma}_{\boldsymbol{\Delta}}=4\left(\boldsymbol{\Delta}_{1100}^{R R}+\boldsymbol{\Delta}_{0011}^{R R}\right)
$$

and the bias term in computed by averaging (52) over the channel variable as per (23).

Codes for $N_{t}=4$ can easily be obtained by appending an appropriate row to the $N_{t}=3$ counterparts. For example, a 4-transmit antenna code based on $\mathbf{X}_{(3)}$ in (47) is given by,

$$
\mathbf{X}_{(4)}=\left[\begin{array}{cccc}
a_{0} & a_{1}^{*} & a_{2}^{*} & 0 \\
-a_{1} & a_{0}^{*} & 0 & -a_{2}^{*} \\
-a_{2} & 0 & a_{0}^{*} & a_{1}^{*} \\
0 & a_{2} & -a_{1} & a_{0}
\end{array}\right]
$$

Due to similarities in the structure of $\mathbf{X}_{(3)}$ and $\mathbf{X}_{(4)}$, a timing estimate for $\mathbf{X}_{(4)}$ can be obtained using $\hat{\epsilon}_{(4)}=\hat{\epsilon}_{(3)}$. The resulting

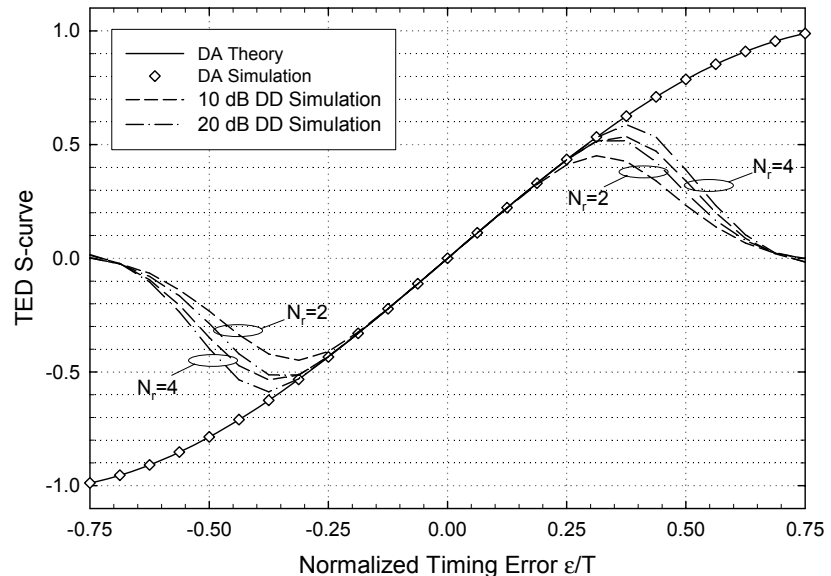

Fig. 2. S-curve for $\hat{\epsilon}_{(4)}$ as applied to $\mathbf{X}_{(4)}$ in (55), $N_{t}=4, N_{r}=2,4$.

$\boldsymbol{\Gamma}_{(4)}$ is given by

$$
\boldsymbol{\Gamma}_{(4)}=2\left[\begin{array}{cccc}
g_{-1}^{\epsilon}-g_{1}^{\epsilon} & -2 g_{0}^{\epsilon} & g_{-3}^{\epsilon}-g_{-1}^{\epsilon} & -2 g_{-2}^{\epsilon} \\
2 g_{0}^{\epsilon} & g_{-1}^{\epsilon}-g_{1}^{\epsilon} & 2 g_{-2}^{\epsilon} & g_{-3}^{\epsilon}-g_{-1}^{\epsilon} \\
g_{1}^{\epsilon}-g_{3}^{\epsilon} & -2 g_{2}^{\epsilon} & g_{-1}^{\epsilon}-g_{1}^{\epsilon} & -2 g_{0}^{\epsilon} \\
2 g_{2}^{\epsilon} & g_{1}^{\epsilon}-g_{3}^{\epsilon} & 2 g_{0}^{\epsilon} & g_{-1}^{\epsilon}-g_{1}^{\epsilon}
\end{array}\right]
$$

where S-curve is given by

$$
E\left\{\hat{\epsilon}_{(4)}\right\}=2 \rho_{2}\left(g_{-1}^{\epsilon}-g_{1}^{\epsilon}\right)+E_{\mathbf{H}}\left\{\delta_{\hat{\epsilon}_{(4)}}\right\}
$$

with the TEM bias of

$$
\begin{aligned}
& \delta_{\hat{\epsilon}_{(4)}}=\|\mathbf{H}\|^{-2} 2 \rho_{2} \sum_{j=1}^{N_{r}}\left[2\left(g_{-2}^{\epsilon}-g_{2}^{\epsilon}\right) \Re\left(h_{j 3}^{*} h_{j 2}-h_{j 4}^{*} h_{j 1}\right)\right. \\
& \left.-\left(g_{-1}^{\epsilon}-g_{1}^{\epsilon}-g_{-3}^{\epsilon}+g_{3}^{\epsilon}\right) \Re\left(h_{j 3}^{*} h_{j 1}+h_{j 4}^{*} h_{j 2}\right)\right] \text {. }
\end{aligned}
$$

The variance for $\hat{\epsilon}_{(4)}$ can be computed using (53) and (54), with the components $\boldsymbol{\Phi}_{i j m n}^{R R}$ and $\boldsymbol{\Delta}_{i j m n}^{R R}$ appropriately modified for the $N_{t}=4$ code

\section{TED PROPERTIES}

We now evaluate the properties of TED $\hat{\epsilon}_{(4)}=\hat{\epsilon}_{(3)}$ given by (48) as applied to $\mathbf{X}_{(4)}$, beginning with the S-curve shown in Fig. 2. The analytical results were obtained using (57), where the expectation of $\delta_{\hat{\epsilon}_{(4)}}$ was computed numerically over $10^{4}$ instances of $\mathbf{H}$. We verify the analytical results via simulation, where the data was sampled at a fixed, uncompensated timing error $\epsilon$ and the resulting TED output $\hat{\epsilon}$ in (48) averaged over all code blocks transmitted. Finally, the effect of data decision errors was evaluated by replacing the information symbols in the data-aided (DA)-TED in (48) by the corresponding decisions, resulting in a decision-directed (DD)-TED. The system SNR $E_{s} / N_{0}$ was set to 10 and $20 \mathrm{~dB}$.

In the case of DA-TED, the simulated results follow the theoretical expressions very closely. Examining the DD Scurve, we note that incorrect data decisions reduce the linear region to approximately $|\epsilon / T|=0.25$ and $|\epsilon / T|=0.35$ for $N_{r}=2$ and $N_{r}=4$, respectively. One notes that in the case DD operation, the output of the TED will be periodic for increasing $\epsilon$. As shown in [16], for the case of single antenna 


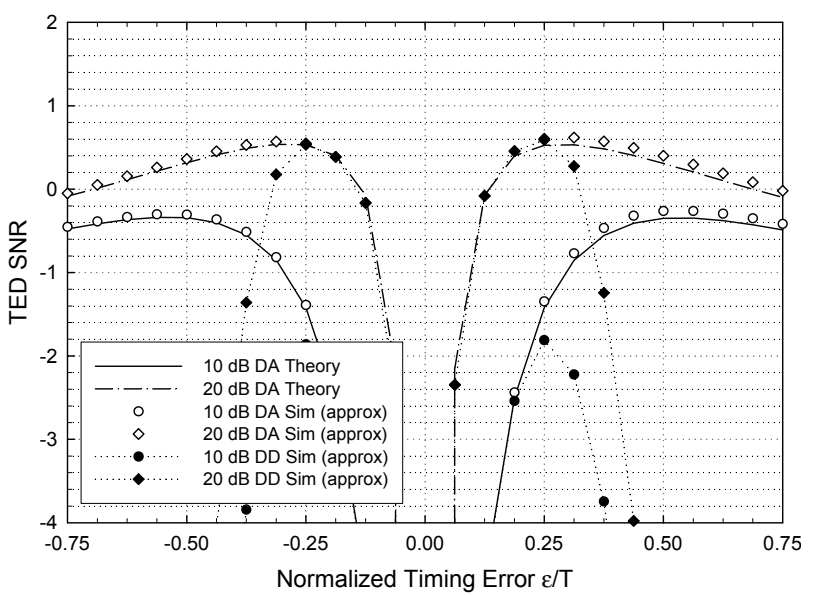

Fig. 3. SNR for TED $\hat{\epsilon}_{(4)}$ as applied to $\mathbf{X}_{(4)}$ in (55), $N_{t}=4, N_{r}=2$.

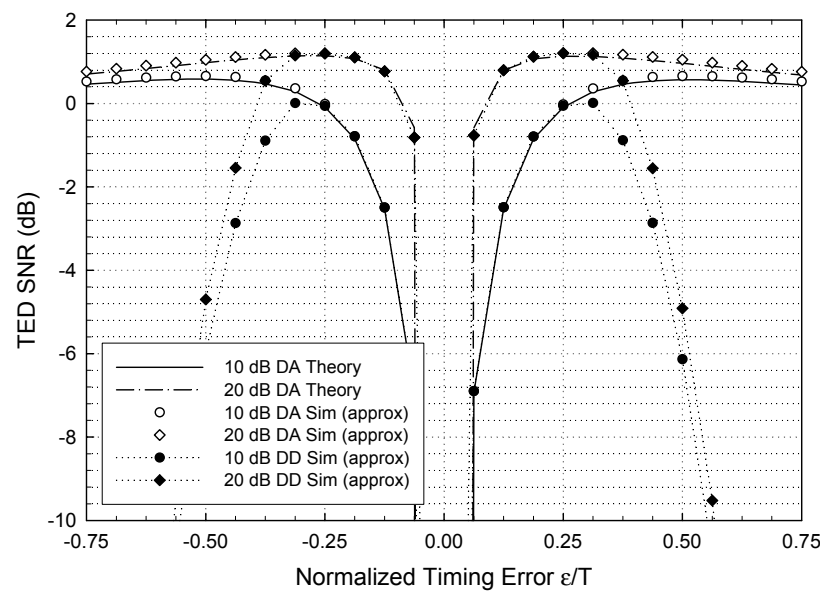

Fig. 4. SNR for TED $\hat{\epsilon}_{(4)}$ as applied to $\mathbf{X}_{(4)}$ in (55), $N_{t}=4, N_{r}=4$.

systems where the decisions are made every symbol interval, the S-curve will exhibit periodicity of $T$. In contrast, in the case of OSTBC systems, block decoding at the receiver will result in TED periodicity of $N_{c} T$.

Figs. 3 and 4 show the TED SNR for $\hat{\epsilon}_{(4)}$. The theoretical results were obtained via (41), with the variance term computed by (40) with (53) and (54), and averaged over $10^{4}$ instances of $\mathbf{H}$. Similarly to the S-curve results, we verify the analytical results by means of simulation, using the approximated TED SNR in (42), with the error variance calculated by averaging $(\epsilon-\hat{\epsilon})^{2}$ over all code blocks transmitted. Once again, DA and $\mathrm{DD}\left(E_{s} / N_{0}=10\right.$ and $\left.20 \mathrm{~dB}\right)$ modes were used.

We note that for moderate $E_{s} / N_{0}$, the TED SNR peaks at a normalized timing error of below $|\epsilon / T|=0.25$, which falls in the operating region of the timing loop. For large timing offset, the estimation variance is large, reducing the TED SNR. This suggests that the loop filter bandwidth should be reduced for low SNR.

Finally, we note that the property being evaluated is the TED output SNR, which constitutes the input SNR of the timing loop. Thus, the TED SNR will be significantly increased by the averaging operation by virtue of the loop filter and the threshold device, described in Section V. Specifically, for a loop that processes $N_{\text {eff }}$ inputs between timing corrections, the TEM error variance will be reduced by approximately $N_{\text {eff }}$, which is equivalent to an increase in the loop SNR of $10 \log _{10} N_{\text {eff }} \mathrm{dB}^{7}$. Thus, for a loop with high $N_{\text {eff }}$, as will be considered in Section V, even a small or moderate TED SNR will result in high loop SNR, allowing for good system performance.

\section{Simulation Results}

We now present simulation results evaluating the timing error tracking ${ }^{8}$ performance of the TEDs proposed in Section III in a system with QPSK-modulated data transmitted over a frequency-flat Rayleigh fading channel. Specifically, we consider the receiver depicted in Fig. 1 with a timing loop employing TED $\hat{\epsilon}_{(3)}$ applied to codes $\mathbf{X}_{(3)}$ and $\mathbf{X}_{(4)}{ }^{9}$. Sections $\mathrm{V}-\mathrm{A}$ and V-B evaluate the SER performance as a function of system SNR and timing drift bandwidth, respectively. While the discussion in Sections III and IV assumed a receiver with perfect channel knowledge, we also examine the effects of channel estimation errors on the system performance. To that end we present results for CSIR as well as pilot-symbol assisted modulation (PSAM)-based receivers.

The data was encoded using $\mathbf{X}_{(3)}$ and $\mathbf{X}_{(4)}$, given by (47) and (55), respectively. The resulting data streams were passed through a RRC filter with a rolloff of $\beta_{M F}=0.35$. The fading was assumed to be Rayleigh distributed, with the autocorrelation given by (2) with a known normalized Doppler frequency of $f_{D} T=0.01$. In the simulations, we used a modified Jakes model in [17]. Since, as stated in Section II, the channel response was assumed to be quasi-static, the channel gains generated by the simulator in [17] were held constant for the duration of an OSTBC block and subsequently updated for the next block according to the correlation defined by (2).

As the focus of the simulations is on timing error tracking, we assume that the receiver has performed coarse timing acquisition, which would typically be done using a training sequence. The timing drift was simulated by perturbing the sampling phase $\tau$. In order to add a random component to the timing drift, the time between timing slips, measured in symbol intervals and denoted by $N_{\tau}$, was modeled by a Gaussian random variable, with a mean of $\bar{N}_{\tau}$ and with a variance of $\sigma_{N_{\tau}}^{2}=0.1 \bar{N}_{\tau}$.

The drift direction was random and equiprobable, with a resolution of $T / 16$. We note that since all aspects of the drift model were assumed unknown to the receiver, the step sizes for the drift and the correction were chosen independently, with the latter set to $T / 8 .{ }^{10}$

\footnotetext{
${ }^{7}$ If the loop filter was in the form of a true integrator, the gain in TED SNR would be $10 \log _{10} N_{\text {eff }} \mathrm{dB}$. In the case of a low pass filter considered in Section V, this effective TED SNR gain is only an approximation.

${ }^{8}$ Receiver incorporating a feedback timing loop is used throughout the simulations. The problem of timing acquisition, where a feed-forward configuration exhibiting fast recovery is preferred [16], is not considered in this paper.

${ }^{9}$ The performance of TED $\hat{\epsilon}_{(2)}$ was described in [8].

${ }^{10}$ Unlike other timing offset models employed (see, for example [18]), the discrete nature of the model considered here may result in residual timing errors. The authors have found, however, that the magnitude of such errors is negligible. Results equivalent to those presented here were also obtained using simulations with sampling resolutions of $T / 32$ and $T / 8$.
} 
The resulting mean timing error bandwidth, normalized to the symbol duration $T$, is given by

$$
\bar{B}_{\tau} T=\frac{T / 16}{\bar{N}_{\tau} T}=\frac{1}{16 \bar{N}_{\tau}} .
$$

We note that modern day clocks, such as temperature compensated crystal oscillators (TCXOs), exhibit a frequencytemperature stability of well under 10 parts per million (ppm) [19]. Using the notation of (59), this corresponds to $\bar{B}_{\tau} T<10^{-5}$. The simulations results presented here consider a much higher $\left(\bar{B}_{\tau} T=10^{-4}\right.$ in Section V-A and up to $\bar{B}_{\tau} T=10^{-3}$ in Section V-B) in order to ensure frequent timing corrections and to maintain a safe margin in relation to hardware specifications.

In the case of results for a receiver with imperfect channel knowledge, we consider a PSAM-based channel estimation approach based on the method presented in [4]. Orthogonal pilot blocks were inserted following $N_{b}=3$ data code blocks, each spanning $N_{c}=4$ symbol intervals. The resulting pilot spacing of $L_{f}=16$ is adequate for the estimation of channel with normalized Doppler frequency up to $f_{D} T=0.03$. The channel state for the data portion of the frames was obtained by interpolating channel estimates from $K=9$ pilot blocks, following which the data was decoded according to (10). In all of the reported results, the system SNR takes into account the overhead due to pilot insertion. Specifically, we define SNR as the average energy $\bar{E}_{s}$ per information symbol, that is $\bar{E}_{s}$ is given by the ratio of the total energy transmitted to the number of information bearing symbols in each frame.

Timing estimation was done using the TED $\hat{\epsilon}_{(3)}$, which as mentioned previously, is applicable to codes $\mathbf{X}_{(3)}$ and $\mathbf{X}_{(4)}$. Since the focus of the investigation is the tracking performance of the detector, the timing estimation was done without the knowledge of the data symbols at the receiver. Hence the data symbols $a_{m}$ were replaced by the corresponding decisions $\hat{a}_{m}$. The TED output was filtered according to

$$
\hat{\epsilon}_{l}^{\prime}=\alpha \hat{\epsilon}_{l-1}^{\prime}+(1-\alpha) \hat{\epsilon}_{l}
$$

with $\alpha=0.9$. If the filtered timing measurement $\hat{\epsilon}_{l}^{\prime}$ exceeded a threshold value $\epsilon_{t h}=0.25$, the timing correction $\hat{\tau}_{l}$ was adjusted by $T / 8$ depending on the polarity of the error estimate. In practice, the error correction procedure can be implemented by means of a bank of polyphase filters [20].

\section{A. SER Performance}

Figs. 5 and 6 show QPSK SER plots for the 3- and 4transmit antenna codes. The figures include TED tracking performance for CSIR and PSAM receivers. Also provided are two reference curves: ideal timing with perfect channel knowledge, and ideal timing with PSAM channel estimation. The mean timing drift bandwidth was fixed to $\bar{B}_{\tau} T=1 \times 10^{-4}$.

The results demonstrate that the receiver is able to track the timing variation with a performance drop resulting from the timing synchronization of less than $0.3 \mathrm{~dB}$. Similar results hold for PSAM-based receivers. By examining the reference curves, it is clear that for most part, the performance loss is due to channel estimation. For the case of QPSK system considered here, the losses due to timing synchronization are

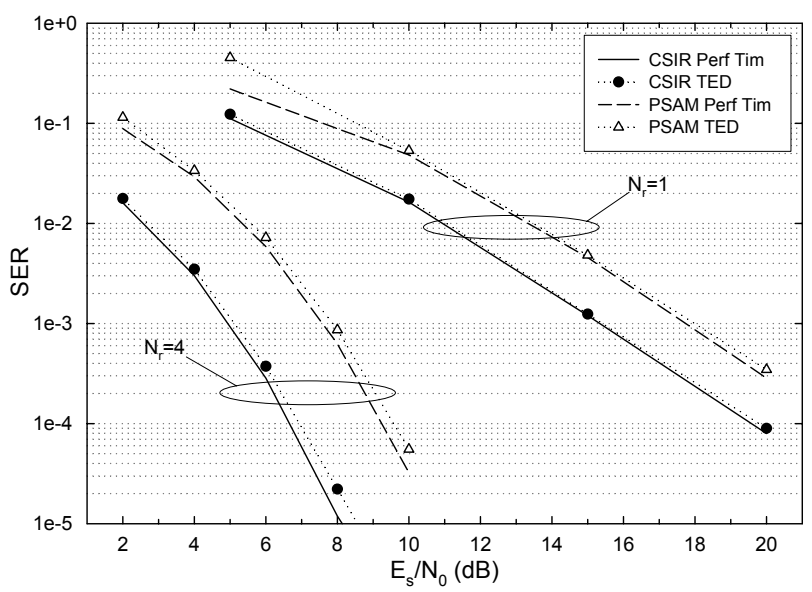

Fig. 5. QPSK SER for $N_{t}=3$ code $\mathbf{X}_{(3)}$ in (47).

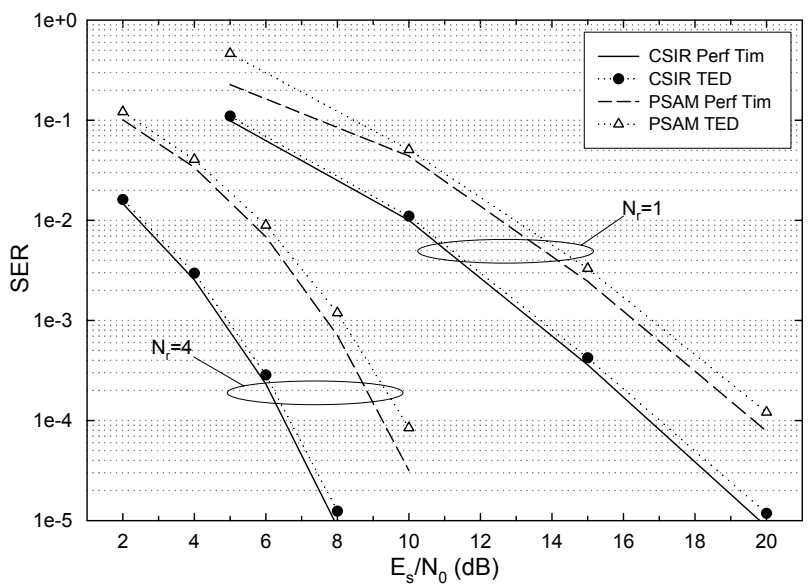

Fig. 6. QPSK SER for $N_{t}=4$ code $\mathbf{X}_{(4)}$ in (55).

very low and leave no practical room for improvement with ML techniques ${ }^{11}$. Further research is required to determine the suitability of the proposed methods for higher order constellations.

\section{B. Performance as a Function of Timing Bandwidth}

We now consider the SER performance for varying timing drift rate. Figs. 7 and 8 show SER as a function of $\bar{B}_{\tau} T$ for $N_{t}=3$ and $N_{t}=4$ codes with $N_{r}=2$ receiver operating at $\bar{E}_{s} / N_{0}=8 \mathrm{~dB}$ and $10 \mathrm{~dB}$. Both CSIR and PSAM ( $K=9$, $\left.L_{f}=16\right)$ cases are shown.

In the case of CSIR, the system is able to track timing up to $\bar{B}_{\tau} T=10^{-3}$, which is over two orders of magnitude greater than the requirements of present day TXCOs [19]. For PSAM-based channel estimation, the range decreases just below $\bar{B}_{\tau} T=10^{-3}$. The difference in performance of CSIR and PSAM receivers can be attributed to the effect of channel estimation error on the TED output, as well as the delay associated with PSAM interpolation resulting in data decoding delay and thus outdated timing information. This effect is more pronounced for faster timing drift, as seen in Figs. 7 and 8.

\footnotetext{
${ }^{11}$ Similar results were observed for BPSK signaling in [11].
} 


$$
\begin{aligned}
E^{\mathbf{H}}\left\{a_{n} s_{m}\right\} & =\|\mathbf{H}\|^{-2} \operatorname{tr}\left\{\mathbf{A}_{m} \mathbf{G}_{\epsilon}^{H}\left[E\left\{\Re\left(a_{n}^{R} \mathbf{X}^{H}\right)+j \Re\left(a_{n}^{I} \mathbf{X}^{H}\right)\right\} \Re\left(\mathbf{H}^{H} \mathbf{H}\right)-E\left\{\Im\left(a_{n}^{R} \mathbf{X}^{H}\right)+j \Im\left(a_{n}^{I} \mathbf{X}^{H}\right)\right\} \Im\left(\mathbf{H}^{H} \mathbf{H}\right)\right]\right\} \\
& -j\|\mathbf{H}\|^{-2} \operatorname{tr}\left\{\mathbf{B}_{m} \mathbf{G}_{\epsilon}^{H}\left[E\left\{\Im\left(a_{n}^{R} \mathbf{X}^{H}\right)+j \Im\left(a_{n}^{I} \mathbf{X}^{H}\right)\right\} \Re\left(\mathbf{H}^{H} \mathbf{H}\right)+E\left\{\Re\left(a_{n}^{R} \mathbf{X}^{H}\right)+j \Re\left(a_{n}^{I} \mathbf{X}^{H}\right)\right\} \Im\left(\mathbf{H}^{H} \mathbf{H}\right)\right]\right\}
\end{aligned}
$$

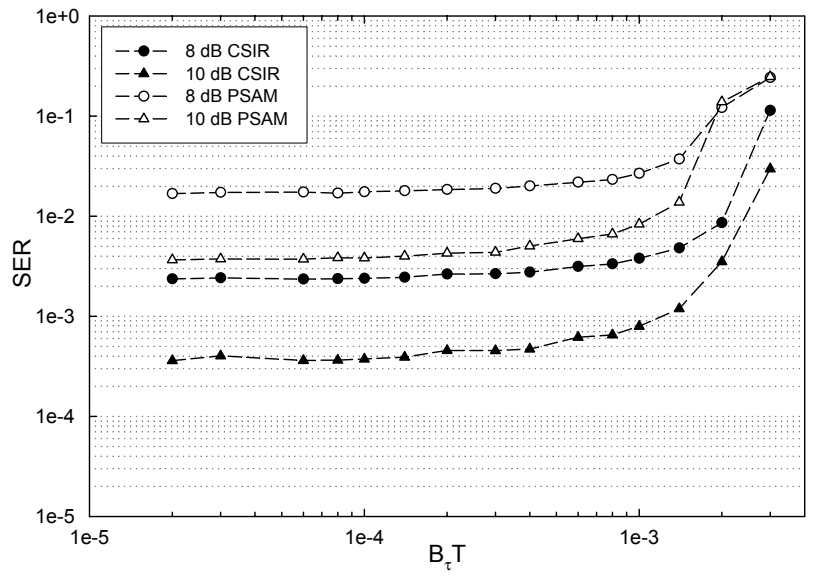

Fig. 7. SER vs $\bar{B}_{\tau} T$ for $N_{t}=3$ code $\mathbf{X}_{(3)}$ in (47).

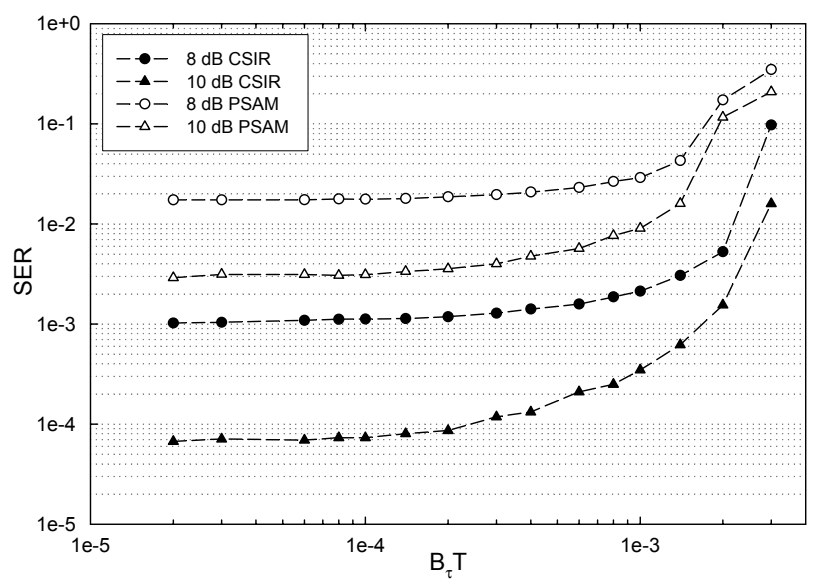

Fig. 8. SER vs $\bar{B}_{\tau} T$ for $N_{t}=4$ code $\mathbf{X}_{(4)}$ in (55).

\section{CONCLUSION}

We have described a method for the design of low complexity TEDs for a general OSTBC system. A set of sufficient conditions for timing estimate robust to channel fading was presented, along with examples of TEDs for 2-, 3- and 4transmit antenna codes. In addition to the S-curve of the TEDs, analytical expressions for the estimation error variance and TED SNR were obtained. We have evaluated the performance of the TEDs in timing loop of a receiver operating in a tracking mode, for both CSIR and PSAM systems. We have shown that the SER performance exhibits negligible degradation due to timing synchronization and that the proposed TEDs are able to track timing drift up to approximately $\bar{B}_{\tau} T=10^{-3}$ with a reduction in range due to PSAM.

\section{APPENDiX A \\ TED DESIGN CONDITIONS}

This section gives details on the derivation of (14) and (15). We begin by noting that using (7) and (10), the decision variables $s_{m, l}$ can be expressed as

$$
\begin{aligned}
s_{m, l}=\left\|\mathbf{H}_{l}\right\|^{-2} & \operatorname{tr}\left\{\mathbf{A}_{m} \Re\left(\mathbf{X}_{\epsilon, l}^{H} \mathbf{H}_{l}^{H} \mathbf{H}_{l}\right)\right. \\
& \left.-j \mathbf{B}_{m} \Im\left(\mathbf{X}_{\epsilon, l}^{H} \mathbf{H}_{l}^{H} \mathbf{H}_{l}\right)\right\}+\eta^{\prime}
\end{aligned}
$$

where we have used the fact that $\operatorname{tr}(\mathbf{A B})=\operatorname{tr}(\mathbf{B A})$ and $\Re(\operatorname{tr}(\cdot))=\operatorname{tr}(\Re(\cdot)), \Im(\operatorname{tr}(\cdot))=\operatorname{tr}(\Im(\cdot))$. The noise term $\eta^{\prime}$ in (61) is given by

$$
\eta^{\prime}=\left\|\mathbf{H}_{l}\right\|^{-2} \operatorname{tr}\left\{\Re\left(\mathbf{N}_{l}^{H} \mathbf{H}_{l}^{H} \mathbf{A}_{m}\right)-j \Im\left(\mathbf{N}_{l}^{H} \mathbf{H}_{l}^{H} \mathbf{B}_{m}\right)\right\} .
$$

Substituting (8) into (61) we obtain

$$
\begin{aligned}
E^{\mathbf{H}}\left\{a_{n, l} s_{m, l}\right\} & = \\
\left\|\mathbf{H}_{l}\right\|^{-2} & \operatorname{tr}\left\{\mathbf{A}_{m} \sum_{n^{\prime}} \mathbf{G}_{\epsilon, n^{\prime}}^{H} E\left\{a_{n, l} \Re\left(\mathbf{X}_{l+n^{\prime}}^{H} \mathbf{H}_{l}^{H} \mathbf{H}_{l}\right)\right\}\right. \\
& \left.-j \mathbf{B}_{m} \sum_{n^{\prime}} \mathbf{G}_{\epsilon, n^{\prime}}^{H} E\left\{a_{n, l} \Im\left(\mathbf{X}_{l+n^{\prime}}^{H} \mathbf{H}_{l}^{H} \mathbf{H}_{l}\right)\right\}\right\} .
\end{aligned}
$$

Since the data symbols $a_{n, l}$ are used to encode only the $l$ th block $\mathbf{X}_{l}, \quad E\left\{a_{n, l} \Re\left(\mathbf{X}_{l+n^{\prime}}^{H} \mathbf{H}_{l}^{H} \mathbf{H}_{l}\right)\right\}=$ $E\left\{a_{n, l} \Im\left(\mathbf{X}_{l+n^{\prime}}^{H} \mathbf{H}_{l}^{H} \mathbf{H}_{l}\right)\right\}=0$ for $n^{\prime} \neq 0$. Therefore (63) simplifies to

$$
\begin{aligned}
E^{\mathbf{H}}\left\{a_{n, l} s_{m, l}\right\} & = \\
\left\|\mathbf{H}_{l}\right\|^{-2} & \operatorname{tr}\left\{\mathbf{A}_{m} \mathbf{G}_{\epsilon}^{H} E^{\mathbf{H}}\left\{a_{n, l} \Re\left(\mathbf{X}_{l}^{H} \mathbf{H}_{l}^{H} \mathbf{H}_{l}\right)\right\}\right. \\
& \left.-j \mathbf{B}_{m} \mathbf{G}_{\epsilon}^{H} E^{\mathbf{H}}\left\{a_{n, l} \Im\left(\mathbf{X}_{l}^{H} \mathbf{H}_{l}^{H} \mathbf{H}_{l}\right)\right\}\right\}
\end{aligned}
$$

where we have dropped the zero-valued subscript $n^{\prime}$ from $\mathbf{G}_{\epsilon, n^{\prime}}$ for notational convenience. In addition, we can now remove the code block index $l$, with the understanding that $a_{n}, s_{m}, \mathbf{X}$ and $\mathbf{H}$ refer to the block used for the estimation of the timing error. Using the fact that

$$
\Re\{\mathbf{A B}\}=\Re\{\mathbf{A}\} \Re\{\mathbf{B}\}-\Im\{\mathbf{A}\} \Im\{\mathbf{B}\}
$$

and

$$
\Im\{\mathbf{A B}\}=\Im\{\mathbf{A}\} \Re\{\mathbf{B}\}+\Re\{\mathbf{A}\} \Im\{\mathbf{B}\}
$$

we now expand (64) to give (67).

Using (1), and assuming independent data with independent real and imaginary components, one can show that

$$
\begin{array}{r}
E\left\{a_{n}^{R} \mathbf{X}^{H}\right\} \triangleq \rho_{2} \mathbf{A}_{n}^{H} \\
E\left\{a_{n}^{I} \mathbf{X}^{H}\right\} \triangleq-j \rho_{2} \mathbf{B}_{n}^{H}
\end{array}
$$

where the constant $\rho_{p}$ is defined by (16). Substituting (68), and noting that $\Re\left(j \rho_{2} \mathbf{B}_{n}^{H}\right)=0$ and $\Im\left(\rho_{2} \mathbf{A}_{n}^{H}\right)=0$, one can simplify (67) to the final result given by (14).

The derivation of $E\left\{a_{n}^{l *} s_{m}^{l}\right\}$ follows the same line of reasoning as for $E\left\{a_{n}^{l} s_{m}^{l}\right\}$, resulting in the solution given in (15). See [11] for more details. 


$$
\begin{aligned}
& E^{\mathbf{H}}\left\{a_{i}^{R} a_{j}^{R} s_{m}^{R} s_{n}^{R}\right\}=\|\mathbf{H}\|^{-4} E^{\mathbf{H}}\left\{a_{i}^{R} a_{j}^{R} \times\right. \\
& \operatorname{tr}\left[\sum_{l} \mathbf{A}_{m} \mathbf{G}_{\epsilon, l}^{H} \Re\left(\mathbf{X}_{l}^{H} \mathbf{H}^{H} \mathbf{H}\right) \otimes \sum_{l^{\prime}} \mathbf{A}_{n} \mathbf{G}_{\epsilon, l^{\prime}}^{H} \Re\left(\mathbf{X}_{l^{\prime}}^{H} \mathbf{H}^{H} \mathbf{H}\right)+\mathbf{A}_{m} \Re\left(\mathbf{N}^{H} \mathbf{H}\right) \otimes \mathbf{A}_{n} \Re\left(\mathbf{N}^{H} \mathbf{H}\right)\right. \\
& \left.\left.+\sum_{l} \mathbf{A}_{m} \mathbf{G}_{\epsilon, l}^{H} \Re\left(\mathbf{X}_{l}^{H} \mathbf{H}^{H} \mathbf{H}\right) \otimes \mathbf{A}_{n} \Re\left(\mathbf{N}^{H} \mathbf{H}\right)+\mathbf{A}_{m} \Re\left(\mathbf{N}^{H} \mathbf{H}\right) \otimes \sum_{l} \mathbf{A}_{n} \mathbf{G}_{\epsilon, l}^{H} \Re\left(\mathbf{X}_{l}^{H} \mathbf{H}^{H} \mathbf{H}\right)\right]\right\} . \\
& E^{\mathbf{H}}\left\{\left(a_{i}^{R}\right)^{2} \operatorname{tr}\left(\mathbf{\Psi}_{X}^{R R}\right)\right\}=\rho_{2}^{2} \operatorname{tr}\left\{\sum_{l} \sum_{k}\left(\mathbf{A}_{m} \mathbf{G}_{\epsilon, l}^{H} \otimes \mathbf{A}_{n} \mathbf{G}_{\epsilon, l}^{H}\right)\left[\left(\mathbf{A}_{k}^{H} \otimes \mathbf{A}_{k}^{H}\right) \boldsymbol{\Omega}_{\mathrm{RR}}+\left(\mathbf{B}_{k}^{H} \otimes \mathbf{B}_{k}^{H}\right) \boldsymbol{\Omega}_{\mathrm{II}}\right]\right\} \\
& +\operatorname{tr}\left\{\left(\mathbf{A}_{m} \mathbf{G}_{\epsilon, 0}^{H} \otimes \mathbf{A}_{n} \mathbf{G}_{\epsilon, 0}^{H}\right)\left[\left(\rho_{4}-\rho_{2}^{2}\right)\left(\mathbf{A}_{i}^{H} \otimes \mathbf{A}_{i}^{H}\right) \boldsymbol{\Omega}_{\mathrm{RR}}+\left(\rho_{2}^{\prime}-\rho_{2}^{2}\right)\left(\mathbf{B}_{i}^{H} \otimes \mathbf{B}_{i}^{H}\right) \boldsymbol{\Omega}_{\mathrm{II}}\right]\right\} \\
& E^{\mathbf{H}}\left\{\left(a_{i}^{R}\right)^{2} \operatorname{tr}\left(\mathbf{\Psi}_{N}^{R R}\right)\right\}=\rho_{2} \operatorname{tr}\left\{( \mathbf { A } _ { m } \otimes \mathbf { A } _ { n } ) E ^ { \mathbf { H } } \left\{\left(\Re\left(\mathbf{N}^{H}\right) \otimes \Re\left(\mathbf{N}^{H}\right)\right) \boldsymbol{\Omega}_{\mathrm{RR}}^{\prime}+\left(\Im\left(\mathbf{N}^{H}\right) \otimes \Im\left(\mathbf{N}^{H}\right)\right) \boldsymbol{\Omega}_{\mathrm{II}}^{\prime}\right.\right. \\
& \left.\left.-\left(\Re\left(\mathbf{N}^{H}\right) \otimes \Im\left(\mathbf{N}^{H}\right)\right) \boldsymbol{\Omega}_{\mathrm{RI}}^{\prime}-\left(\Im\left(\mathbf{N}^{H}\right) \otimes \Re\left(\mathbf{N}^{H}\right)\right) \boldsymbol{\Omega}_{\mathrm{IR}}^{\prime}\right\}\right\}
\end{aligned}
$$

\section{APPENDIX B \\ TED VARIANCE}

We present the derivations of (27), (34) and (37) ${ }^{12}$. We begin by considering $E^{\mathbf{H}}\left\{a_{i}^{R} a_{j}^{R} s_{m}^{R} s_{n}^{R}\right\}$, as defined by (26). Using the fact that $\operatorname{tr}(\mathbf{A}) \operatorname{tr}(\mathbf{B})=\operatorname{tr}(\mathbf{A} \otimes \mathbf{B})$ [21, p.250], we can express (26) by

$$
\begin{aligned}
E^{\mathbf{H}}\left\{a_{i}^{R} a_{j}^{R} s_{m}^{R} s_{n}^{R}\right\}=\|\mathbf{H}\|^{-4} E^{\mathbf{H}}\left\{a_{i}^{R} a_{j}^{R} \times\right. \\
\left.\operatorname{tr}\left(\mathbf{A}_{m} \Re\left(\mathbf{Y}^{H} \mathbf{H}\right) \otimes \mathbf{A}_{n} \Re\left(\mathbf{Y}^{H} \mathbf{H}\right)\right)\right\} .
\end{aligned}
$$

Using (7) along with the distributive property [21, p.243] of the Kronecker product, and assuming mutually independent data and noise, we expand (69), obtaining (70). We note that the expectation of the last two arguments of the trace operator in (70) is zero since the noise is assumed to be zero-mean and independent of data $a_{i}$ and $\mathbf{X}_{l}$. Thus, after some manipulation we can simplify (70) to obtain

$$
E^{\mathbf{H}}\left\{a_{i}^{R} a_{j}^{R} s_{m}^{R} s_{n}^{R}\right\}=\|\mathbf{H}\|^{-4} E^{\mathbf{H}}\left\{a_{i}^{R} a_{j}^{R} \operatorname{tr}\left(\mathbf{\Psi}_{X}^{R R}+\mathbf{\Psi}_{N}^{R R}\right)\right\}
$$

where $\Psi_{X}^{R R}$ and $\boldsymbol{\Psi}_{N}^{R R}$ correspond to the data and noise components of the trace argument in (70), respectively, and are defined by

$$
\begin{aligned}
\mathbf{\Psi}_{X}^{R R}= & \sum_{l} \mathbf{A}_{m} \mathbf{G}_{\epsilon, l}^{H} \Re\left(\mathbf{X}_{l}^{H} \mathbf{H}^{H} \mathbf{H}\right) \otimes \\
& \sum_{l^{\prime}} \mathbf{A}_{n} \mathbf{G}_{\epsilon, l^{\prime}}^{H} \Re\left(\mathbf{X}_{l^{\prime}}^{H} \mathbf{H}^{H} \mathbf{H}\right)
\end{aligned}
$$

and

$$
\mathbf{\Psi}_{N}^{R R}=\mathbf{A}_{m} \Re\left(\mathbf{N}^{H} \mathbf{H}\right) \otimes \mathbf{A}_{n} \Re\left(\mathbf{N}^{H} \mathbf{H}\right) .
$$

Examining (72), we recall that the data blocks are given by (1) and note that the data symbols $a_{i}$ and $a_{j}$ in (71) belong to block $l=0$. Since data symbols are zero mean, the summation in (72) will contribute only the $l=l^{\prime}$ terms, with the remaining components vanishing with the expectation operator. Thus, using the fact that $\mathbf{A B} \otimes \mathbf{C D}=(\mathbf{A} \otimes \mathbf{C})(\mathbf{B} \otimes \mathbf{D})$ [21, p.244], we obtain

$$
\begin{array}{r}
E^{\mathbf{H}}\left\{a_{i}^{R} a_{j}^{R} \operatorname{tr}\left(\mathbf{\Psi}_{X}^{R R}\right)\right\}=\operatorname{tr}\left\{\sum_{l}\left(\mathbf{A}_{m} \mathbf{G}_{\epsilon, l}^{H} \otimes \mathbf{A}_{n} \mathbf{G}_{\epsilon, l}^{H}\right) \times\right. \\
\left.E^{\mathbf{H}}\left\{a_{i}^{R} a_{j}^{R} \Re\left(\mathbf{X}_{l}^{H} \mathbf{H}^{H} \mathbf{H}\right) \otimes \Re\left(\mathbf{X}_{l}^{H} \mathbf{H}^{H} \mathbf{H}\right)\right\}\right\}
\end{array}
$$

${ }^{12} \mathrm{~A}$ more detailed derivation has been presented in [11]. which can be further expanded using (65) and (66) resulting in

$$
\begin{aligned}
& E^{\mathbf{H}}\left\{a_{i}^{R} a_{j}^{R} \operatorname{tr}\left(\mathbf{\Psi}_{X}^{R R}\right)\right\}= \\
& \operatorname{tr}\left\{\sum _ { l } ( \mathbf { A } _ { m } \mathbf { G } _ { \epsilon , l } ^ { H } \otimes \mathbf { A } _ { n } \mathbf { G } _ { \epsilon , l } ^ { H } ) E ^ { \mathbf { H } } \left\{a_{i}^{R} a_{j}^{R} \times\right.\right. \\
& \quad\left[\left(\Re\left(\mathbf{X}_{l}^{H}\right) \otimes \Re\left(\mathbf{X}_{l}^{H}\right)\right) \boldsymbol{\Omega}_{\mathrm{RR}}+\left(\Im\left(\mathbf{X}_{l}^{H}\right) \otimes \Im\left(\mathbf{X}_{l}^{H}\right)\right) \boldsymbol{\Omega}_{\mathrm{II}}\right. \\
& \left.\left.\left.\quad-\left(\Re\left(\mathbf{X}_{l}^{H}\right) \otimes \Im\left(\mathbf{X}_{l}^{H}\right)\right) \boldsymbol{\Omega}_{\mathrm{RI}}-\left(\Im\left(\mathbf{X}_{l}^{H}\right) \otimes \Re\left(\mathbf{X}_{l}^{H}\right)\right) \boldsymbol{\Omega}_{\mathrm{IR}}\right]\right\}\right\}
\end{aligned}
$$

with $\boldsymbol{\Omega}_{\mathrm{RR}}, \boldsymbol{\Omega}_{\mathrm{II}}, \boldsymbol{\Omega}_{\mathrm{RI}}, \boldsymbol{\Omega}_{\mathrm{IR}}$ defined by (32). Substituting (1), and assuming independent and zero mean data components, once can show [11] that for $i \neq j$,

$$
\begin{aligned}
& E^{\mathbf{H}}\left\{a_{i}^{R} a_{j}^{R} \operatorname{tr}\left(\mathbf{\Psi}_{X}^{R R}\right)\right\}=\rho_{2}^{2} \operatorname{tr}\left\{\left(\mathbf{A}_{m} \mathbf{G}_{\epsilon, 0}^{H} \otimes \mathbf{A}_{n} \mathbf{G}_{\epsilon, 0}^{H}\right) \times\right. \\
& \left.\left(\mathbf{A}_{j}^{H} \otimes \mathbf{A}_{i}^{H}+\mathbf{A}_{i}^{H} \otimes \mathbf{A}_{j}^{H}\right) \boldsymbol{\Omega}_{\mathrm{RR}}\right\}
\end{aligned}
$$

where $\rho_{2}$ is defined by (16). Similarly, for $i=j$, one obtains (77), where $\rho_{2}$ and $\rho_{4}$ are defined by (16) and $\rho_{2}^{\prime}$ by (30). We note that the first summation accounts for ISI components over all data blocks and will converge since $\mathbf{G}_{\epsilon, l} \rightarrow 0$ as $l \rightarrow \infty$.

Having solved for the data component in (71), we now solve for the noise term, starting with (73). Since $E\left\{a_{i}^{R}\right\}=0$ and assuming independent data symbols, $E^{\mathbf{H}}\left\{a_{i}^{R} a_{j}^{R} \operatorname{tr}\left(\mathbf{\Psi}_{N}^{R R}\right)\right\}$ will be non-zero only for $i=j$. In such a case, we obtain (78), where $\Omega_{\mathrm{RR}}^{\prime}, \Omega_{\mathrm{II}}^{\prime}, \Omega_{\mathrm{RI}}^{\prime}, \Omega_{\mathrm{IR}}^{\prime}$ are defined (33). Since the real and imaginary noise components are assumed independent and zero-mean, the last two terms in (78) will vanish, resulting in

$E^{\mathbf{H}}\left\{\left(a_{i}^{R}\right)^{2} \operatorname{tr}\left(\mathbf{\Psi}_{N}^{R R}\right)\right\}=\rho_{2} \frac{N_{0}}{2} \operatorname{tr}\left\{\left(\mathbf{A}_{m} \otimes \mathbf{A}_{n}\right) \boldsymbol{\Lambda}_{N}\left(\boldsymbol{\Omega}_{\mathrm{RR}}^{\prime}+\boldsymbol{\Omega}_{\mathrm{II}}^{\prime}\right)\right\}$

where the matrix $\boldsymbol{\Lambda}_{N}$ is defined by (31). Combining (76), (77) and (79) with (71), leads to (26).

The solution to $E^{\mathbf{H}}\left\{a_{i}^{I} a_{j}^{I} s_{m}^{I} s_{n}^{I}\right\}$ can be obtained by following the same methodology as for $E^{\mathbf{H}}\left\{a_{i}^{R} a_{j}^{R} s_{m}^{R} s_{n}^{R}\right\}$ with the solution give by (34). Finally, we consider the term $E^{\mathbf{H}}\left\{a_{i}^{R} a_{j}^{I} s_{m}^{R} s_{n}^{I}\right\}$, which, using the same reasoning as for $E^{\mathbf{H}}\left\{a_{i}^{R} a_{j}^{R} s_{m}^{R} s_{n}^{R}\right\}$ can be expressed as

$$
E^{\mathbf{H}}\left\{a_{i}^{R} a_{j}^{I} s_{m}^{R} s_{n}^{I}\right\}=-\|\mathbf{H}\|^{-4} E^{\mathbf{H}}\left\{a_{i}^{R} a_{j}^{I} \operatorname{tr}\left(\boldsymbol{\Psi}_{X}^{R I}+\mathbf{\Psi}_{N}^{R I}\right)\right\}
$$


where

$$
\begin{aligned}
\mathbf{\Psi}_{X}^{R I}= & \sum_{l} \mathbf{A}_{m} \mathbf{G}_{\epsilon, l}^{H} \Re\left(\mathbf{X}_{l}^{H} \mathbf{H}^{H} \mathbf{H}\right) \otimes \\
& \sum_{l^{\prime}} \mathbf{B}_{n} \mathbf{G}_{\epsilon, l^{\prime}}^{H} \Im\left(\mathbf{X}_{l^{\prime}}^{H} \mathbf{H}^{H} \mathbf{H}\right)
\end{aligned}
$$

and

$$
\mathbf{\Psi}_{N}^{R I}=\mathbf{A}_{m} \Re\left(\mathbf{N}^{H} \mathbf{H}\right) \otimes \mathbf{B}_{n} \Im\left(\mathbf{N}^{H} \mathbf{H}\right) .
$$

We note that since real and imaginary components of $\mathbf{N}$ are independent, $E^{\mathbf{H}}\left\{a_{i}^{I} a_{i}^{I} \operatorname{tr}\left(\boldsymbol{\Phi}_{N}^{R I}\right)\right\}=0$ and thus we need only consider the first term of (80). One can show that

$$
\begin{aligned}
& E^{\mathbf{H}}\left\{a_{i}^{R} a_{j}^{I} \operatorname{tr}\left(\mathbf{\Psi}_{X}^{R I}\right)\right\}= \\
& -\operatorname{tr}\left\{\sum _ { l } ( \mathbf { A } _ { m } \mathbf { G } _ { \epsilon , l } ^ { H } \otimes \mathbf { B } _ { n } \mathbf { G } _ { \epsilon , l } ^ { H } ) E ^ { \mathbf { H } } \left\{a_{i}^{R} a_{j}^{I} \times\right.\right. \\
& \quad\left[\left(\Re\left(\mathbf{X}_{l}^{H}\right) \otimes \Im\left(\mathbf{X}_{l}^{H}\right)\right) \boldsymbol{\Omega}_{\mathrm{RR}}-\left(\Im\left(\mathbf{X}_{l}^{H}\right) \otimes \Re\left(\mathbf{X}_{l}^{H}\right)\right) \boldsymbol{\Omega}_{\mathrm{II}}\right. \\
& \left.\left.\left.+\left(\Re\left(\mathbf{X}_{l}^{H}\right) \otimes \Re\left(\mathbf{X}_{l}^{H}\right)\right) \boldsymbol{\Omega}_{\mathrm{RI}}-\left(\Im\left(\mathbf{X}_{l}^{H}\right) \otimes \Im\left(\mathbf{X}_{l}^{H}\right)\right) \boldsymbol{\Omega}_{\mathrm{IR}}\right]\right\}\right\} .
\end{aligned}
$$

Substituting (1) and considering the cases for $i=j$ and $i \neq j$ together, following the same line of reasoning as for $E^{\mathbf{H}}\left\{a_{i}^{R} a_{j}^{R} s_{m}^{R} s_{n}^{R}\right\}$, one arrives at the solution given by (37).

\section{REFERENCES}

[1] S. M. Alamouti, "A simple transmit diversity technique for wireless communication," IEEE J. Select. Areas Commun., vol. 16, no. 8, pp. $1451-1458$, Oct. 1998.

[2] V. Tarokh, N. Seshadri, and A. R. Calderbank, "Space-time block coding for wireless communications: performance criterion and code construction," IEEE Trans Inform. Theory, vol. 44, pp. 744-765, Mar. 1998.

[3] V. Tarokh, H. Jafarkhani, and A. R. Calderbank, "Space-time block coding for wireless communications: performance results," IEEE $J$. Select. Areas Commun., vol. 17, pp. 451-460, Mar. 1999.

[4] A. F. Naguib, V. Tarokh, N. Seshadri, and R. Calderbank, "A spacetime coding modem for high-data-rate wireless communications," IEEE J. Select. Areas Commun., vol. 16, no. 8, pp. 1459-1478, Oct. 1998.

[5] Y.-C. Wu, S. C. Chan, and E. Serpedin, "Symbol-timing estimation in space-time coding systems based on orthogonal training sequences," IEEE Trans. Wireless Commun., vol. 4, no. 2, pp. 603-613, Mar. 2005.

[6] K. Rajawat and A. K. Chaturvedi, "A low complexity symbol timing estimator for MIMO systems using two samples per symbol," IEEE Commun. Lett., vol. 10, no. 7, pp. 525-527, July 2006.

[7] K. H. Mueller and M. Muller, "Timing recovery in digital synchronous data receivers," IEEE Trans Commun., vol. COMM-24, pp. 516-531, May 1976.

[8] P. A. Dmochowski and P. J. McLane, "Robust timing epoch tracking for Alamouti space-time coding in flat Rayleigh fading MIMO channels," in Proc. IEEE ICC, pp. 2397-2401, May 2005.

[9] P. A. Dmochowski and P. J. McLane, "On the properties of robust timing error detector for Alamouti space-time coding in flat Rayleigh fading MIMO channels with randomly distributed timing drift," in Proc. IEEE GLOBECOM, pp. 193-198, Nov. 2005.

[10] G. Ganesan and P. Stoica, "Space-time block codes: a maximum SNR approach," IEEE Trans Inform. Theory, vol. 47, no. 4, pp. 1650-1656, May 2001.

[11] P. A. Dmochowski, "Timing synchronization for multiple input multiple output communication system," Ph.D. thesis, Queen's University, Kingston, ON, Canada, 2006. Available at www.ece.queensu.ca/directory/laboratories/qwcl/thesis.html.

[12] R. H. Clarke, "A statistical theory of mobile-radio reception," Bell Syst. TEch. J., pp. 957-1000, July-Aug. 1968.

[13] G. L. Stuber, Principles of Mobile Communications. Kluwer Academic Publishers, 2001.
[14] E. G. Larsson and P. Stoica, Space-Time Block Coding for Wireless Communications. Cambridge University Press, 2003.

[15] B. Vucetic and J. Yuan, Space-Time Coding. John Wiley \& Sons Canada Ltd., 2003.

[16] U. Mengali and A. N. D'Andrea, Synchronization Techniques for Digital Receivers. New York: Plenum Press, 1997.

[17] P. Dent, G. E. Bottomley, and T. Croft, "Jakes fading model revisited," Electron. Lett., vol. 29, no. 3, pp. 1162-1163, June 1993.

[18] K. Shi and E. Serpedin, "Fast timing recovery for linearly and nonlinearly modulated systems," IEEE Trans Veh. Technol., vol. 54, no. 6, pp. 2017-2023, Nov. 2005.

[19] W. Zhou, H. Zhou, Z. Xuan, and W. Zhang, "Comparison among precision temperature compensated crystal oscillators," in Proc. IEEE International Frequency Control Symposium and Exposition, pp. 575579, Aug. 2005.

[20] F. J. Harris and M. Rice, "Multirate digital filters for symbol timing synchronization in software defined radios," IEEE J. Select. Areas Commun., vol. 19, no. 12, pp. 2346-2357, Dec. 2001.

[21] R. A. Horn and C. R. Johnson, Topics in Matrix Analysis. Cambridge University Press, 1991.

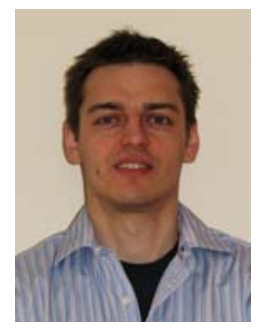

Pawel A. Dmochowski (S'02,M'07) was born in Gdańsk, Poland in 1974. He received a B.A.Sc (Engineering Physics) from the University of British Columbia in 1998, and M.Sc. and Ph.D. degrees from Queen's University, Kingston, Ontario in 2001 and 2006, respectively.

$\mathrm{He}$ is currently a Lecturer in the Faculty of Engineering at Victoria University of Wellington, New Zealand. Previously from 2007 to 2008 , he was an NSERC Visiting Fellow, and subsequently a Research Engineer, at the Communications Research Centre Canada. His research interests include MIMO systems and space-time processing, timing synchronization, channel estimation as well as Cognitive Radio.

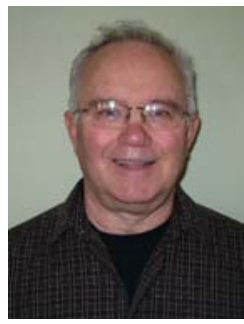

Peter J. McLane (S'68,M'69,SM'80,F'88) was born in Vancouver, BC, Canada, on July 6, 1941. $\mathrm{He}$ received the B.A.Sc. degree from the University of British Columbia, Vancouver, in 1965, the M.S.E.E. degree from the University of Pennsylvania, Philadelphia, in 1966, and the Ph.D. degree from the University of Toronto, Toronto, Ont., Canada, in 1969 .

From 1966 to 1967 , he was a Junior Research Officer with the National Research Council, Ottawa, Ontario, Canada. He joined the Department of Electrical and Computer Engineering, Queen's University, Kingston, Ontario, Canada, in 1969 as an Assistant Professor, and from 1977 to July 1, 2004 he held the rank of Professor. Currently he holds the rank of Professor Emeritus. His research interests are in modulation, coding and signal processing for digital communication systems. He is a former research Trust Leader in Mobile and Satellite Systems for the Telecommunications Research Institute of Ontario (TRIO) and formerly a Major Project Leader in Mobile and Personal Communications for the Canadian Institute of Telecommunications Research (CITR). He is a joint author of Introduction to Trellis-Coded Modulation with Applications (Macmillan, 1991).

Dr. McLane is an IEEE Life Fellow and former chair of the IEEE Communications Society Communication Theory Committee. He is a member-at-large of the Board of Governors of the IEEE Communications Society where he sits on the Awards and Re-Certification Committees. He is a former Associate Editor for the IEEE COMMUNICATIONS MAGAZINE and a former Editor of the IEEE TRANSACTIONS ON COMMUNICATIONS. In addition, he was co-editor of single issues of the IEEE JOURNAL ON SELECTED AREAS IN COMMUNICATIONS and the IEEE COMMUNICATIONS MAGAZINE. He also served as Chairman of the IEEE ComSoc Mini-conference on Communication Theory at Globecom, 1993. He is also a member of the Association of Professional Engineers of Ontario and is listed in American Men and Women in Science. In 1994, he was a joint recipient of the Stentor Telecommunications Research Award. In his graduate studies he held a Ford Foundation Fellowship at the University of Pennsylvania and a National Research Council of Canada Scholarship at the University of Toronto. 